Homology, Homotopy and Applications, vol.10(1), 2008, pp.97-128

\title{
DEFORMATION BICOMPLEX OF MODULE ALGEBRAS
}

\author{
DONALD YAU \\ (communicated by Charles Weibel)
}

\begin{abstract}
The deformation bicomplex of a module algebra over a bialgebra is constructed. It is then applied to study algebraic deformations in which both the module structure and the algebra structure are deformed. The cases of module coalgebras, comodule (co)algebras, and (co)module bialgebras are also considered.
\end{abstract}

\section{Introduction}

Let $H$ be a bialgebra. An $H$-module algebra is an associative algebra $A$ that is also an $H$-module such that the multiplication map on $A$ becomes an $H$-module morphism. This algebraic structure arises often in algebraic topology, quantum groups [11, Chapter V.6], Lie and Hopf algebras theory $[\mathbf{3}, \mathbf{1 5}, \mathbf{1 8}]$, and group representations $[\mathbf{1}$, Chapter 3]. For example, in algebraic topology, the complex cobordism $\mathrm{MU}^{*}(X)$ of a topological space $X$ is an $S$-module algebra, where $S$ is the Landweber-Novikov algebra $[\mathbf{1 3}, \mathbf{1 6}]$ of stable cobordism operations. Likewise, the singular mod $p$ cohomology $\mathrm{H}^{*}(X ; \mathbf{Z} / p)$ of a topological space $X$ is an $\mathcal{A}_{p}$-module algebra, where $\mathcal{A}_{p}$ is the Steenrod algebra associated to the prime $p[4, \mathbf{1 4}]$. More examples of this form can be found in $[\mathbf{2}]$.

The purpose of this paper is twofold:

1. The deformation bicomplex $C_{\mathrm{MA}}^{* *}(A)$ for an $H$-module algebra $A$ is constructed.

2. The deformation bicomplex is used to study algebraic deformations of $A$, where deformations are taken with respect to both the $H$-module structure and the algebra structure on $A$.

The deformation bicomplexes (respectively, tricomplexes) of module coalgebras and comodule (co)algebras (respectively, (co)module bialgebras) are also constructed.

In [20], the author studied algebraic deformations of module algebras, in which only the $H$-module structure is deformed. This paper generalizes [20], which in turn is a generalization of [19]. The current deformation theory of (co)module algebras (respectively, (co)module coalgebras) also generalizes the classical deformation theory of associative algebras [6] (respectively, coalgebras). Moreover, deformation of a (co)module bialgebra is a generalization of the Gerstenhaber-Schack deformation theory of a bialgebra $[\mathbf{7}, \mathbf{8}]$.

Received October 23, 2006; published on January 15, 2008.

2000 Mathematics Subject Classification: 16E40; 16W99.

Key words and phrases: module algebra, module coalgebra, comodule algebra, comodule coalgebra, module bialgebra, comodule bialgebra, deformation.

This article is available at http://intlpress.com/HHA/v10/n1/a5

Copyright (c) 2008, International Press. Permission to copy for private use granted. 
In the deformation bicomplex $C_{\mathrm{MA}}^{* *}(A)$ of an $H$-module algebra $A$, the $(p, q)$-entry is the module $\operatorname{Hom}\left(H^{\otimes q}, \operatorname{Hom}\left(A^{\otimes p}, A\right)\right)$. The 0th row $C_{\mathrm{MA}}^{* 0}(A)$ coincides with the Hochschild cochain complex $\operatorname{Hoch}^{*}(A, A)$ of $A$ with coefficients in itself, which is the deformation complex of $A$ as an associative algebra [6]. In particular, it has a graded Lie bracket [5]. It is not known, however, whether there is a graded Lie bracket on the whole bicomplex $C_{\mathrm{MA}}^{* *}(A)$.

Each higher row

$$
C_{\mathrm{MA}}^{*, q}(A)=\operatorname{Hom}\left(H^{\otimes q}, \operatorname{Hom}\left(A^{\otimes *}, A\right)\right) \quad(q \geqslant 1),
$$

though not a Hochschild cochain complex itself, is isomorphic to one, namely $\operatorname{Hoch}^{*}\left(A, \operatorname{Hom}\left(H^{\otimes q}, A\right)\right)$. Since the module $\operatorname{Hom}\left(H^{\otimes q}, A\right)$ is an associative algebra, it induces an associative product on $\operatorname{Hoch}^{*}\left(A, \operatorname{Hom}\left(H^{\otimes q}, A\right)\right)$, making it into a differential graded associative algebra. In particular, each row in $C_{\mathrm{MA}}^{* *}(A)$ is a differential graded associative algebra. Each higher column

$$
C_{\mathrm{MA}}^{p, *}(A)=\operatorname{Hoch}^{*}\left(H, \operatorname{Hom}\left(A^{\otimes p}, A\right)\right) \quad(p \geqslant 1)
$$

is also a Hochschild cochain complex. However, only the first column $C_{\mathrm{MA}}^{1, *}$ is known to admit a non-trivial associative product, which is induced by the algebra structure on $\operatorname{Hom}(A, A)$.

Here is a summary of the various deformation bicomplexes/tricomplexes constructed in Section 3 and Sections 6-10.

Theorem 1.1. The deformation bicomplex/tricomplex of $A$ is:

1. $C_{\mathrm{MA}}^{* *}(A)=\operatorname{Hoch}^{*}\left(H, \operatorname{Hom}\left(A^{\otimes *}, A\right)\right)$ if $A$ is an $H$-module algebra;

2. $C_{\mathrm{MC}}^{* *}(A)=\operatorname{Hoch}^{*}\left(H, \operatorname{Hom}\left(A, A^{\otimes *}\right)\right)$ if $A$ is an $H$-module coalgebra;

3. $C_{\mathrm{CA}}^{* *}(A)=\operatorname{Hoch}^{*}\left(A, H^{\otimes *} \otimes A\right)$ if $A$ is an $H$-comodule algebra;

4. $C_{\mathrm{CC}}^{* *}(A)=\operatorname{Hom}\left(A, H^{\otimes *} \otimes A^{\otimes *}\right)$ if $A$ is an $H$-comodule coalgebra;

5. $C_{\mathrm{MB}}^{* * *}(A)=\operatorname{Hoch}^{*}\left(H, \operatorname{Hom}\left(A^{\otimes *}, A^{\otimes *}\right)\right)$ if $A$ is an $H$-module bialgebra;

6. $C_{\mathrm{CB}}^{* * *}(A)=\operatorname{Hoch}^{*}\left(A, H^{\otimes *} \otimes A^{\otimes *}\right)$ if $A$ is an $H$-comodule bialgebra.

It should be noted that our deformation bicomplex $C_{\mathrm{MA}}^{* *}(A)$ is different from the Hopf-Hochschild cochain complex $\mathrm{CH}_{\mathrm{Hopf}}^{*}(A, A)$ constructed by Kaygun [12]. The construction of the cochain complex $\mathrm{CH}_{\mathrm{Hopf}}^{*}(A, A)$ is similar to the usual Hochschild cochain complex of $A$, but it also takes into account the $H$-linearity. In particular, if $H$ is the ground field, then $\mathrm{CH}_{\mathrm{Hopf}}^{*}(A, A)$ coincides with the Hochschild cochain complex of $A$. Moreover, $\mathrm{CH}_{\mathrm{Hopf}}^{*}(A, A)$ has the structure of a brace algebra with multiplication [21], which leads to a Gerstenhaber algebra structure on the HopfHochschild cohomology of $A$.

\subsection{Organization}

The rest of this paper is organized as follows.

In Section 2, we fix notations that will be used throughout this paper. The deformation bicomplex $C_{\mathrm{MA}}^{* *}(A)$ of an $H$-module algebra $A$ is constructed in Section 3. In Section 4 , the various $\cup$-products in $C_{\mathrm{MA}}^{* *}(A)$ are constructed.

Algebraic deformations of module algebras are discussed in Section 5. In particular, infinitesimals are properly identified with 2-cocycles in the deformation bicomplex. 
The vanishing of $H^{2}$ of the total complex of the deformation bicomplex implies rigidity, in the sense that every deformation is equivalent to the trivial one.

The constructions and arguments in the module algebra case can be adapted to the cases of module coalgebras, comodule (co)algebras, and (co)module bialgebras. In Sections 6-10, we construct the deformation bicomplexes/tricomplexes and their $\cup$-products for these algebraic structures and state the corresponding deformation results.

\section{Preliminaries}

The purposes of this preliminary section are to fix notations and to recall some basic facts about module algebras and Hochschild cohomology.

\subsection{Notations}

Fix a ground field $K$ once and for all. Modules, linearity, Hom, and $\otimes$ are all meant over $K$, unless otherwise specified. Let $\left(H, \mu_{H}, \Delta_{H}\right)$ be a bialgebra with associative multiplication $\mu_{H}$ and coassociative comultiplication $\Delta_{H}$.

In a coassociative coalgebra $(C, \Delta)$, we use Sweedler's notation $[\mathbf{1 8}]$ for comultiplication,

$$
\Delta^{p}(x)=\sum_{(x)} x_{(1)} \otimes \cdots \otimes x_{(p+1)}
$$

for $p \geqslant 1$. The subscript in $\sum_{(x)}$ will sometimes be omitted.

Given an associative algebra $\left(A, \mu_{A}\right)$, a derivation on $A$ is a linear self-map $\varphi \in$ $\operatorname{End}(A)=\operatorname{Hom}(A, A)$ such that

$$
\varphi(a b)=a \varphi(b)+\varphi(a) b
$$

for all $a, b \in A$. The module of all derivations on $A$ is denoted by $\operatorname{Der}(A)$, which is considered as a submodule of $\operatorname{End}(A)$.

\subsection{Module algebra}

Let $\left(A, \mu_{A}\right)$ be an associative algebra. Then $A$ is said to be an $H$-module algebra if and only if there exists an $H$-module structure $\lambda \in \operatorname{Hom}(H$, $\operatorname{End}(A))$ on $A$ such that $\mu_{A}$ becomes an $H$-module morphism. In other words, $\lambda$ satisfies the following two conditions:

$$
\begin{aligned}
\lambda(x y) & =\lambda(x) \circ \lambda(y), \\
\lambda(x)(a b) & =\sum_{(x)} \lambda\left(x_{(1)}\right)(a) \cdot \lambda\left(x_{(2)}\right)(b),
\end{aligned}
$$

for $x, y \in H$ and $a, b \in A$.

\subsection{Hochschild cohomology}

Let $M$ be an $A$-bimodule. The module of Hochschild $n$-cochains of $A$ with coefficients in $M[\mathbf{9}]$ is defined to be

$$
\operatorname{Hoch}^{n}(A, M) \stackrel{\text { def }}{=} \operatorname{Hom}\left(A^{\otimes n}, M\right) .
$$


The coboundary map

$$
\delta_{h}^{n}: \operatorname{Hoch}^{n}(A, M) \rightarrow \operatorname{Hoch}^{n+1}(A, M)
$$

is given by the alternating sum

$$
\delta_{h}^{n}=\sum_{i=0}^{n+1}(-1)^{i} \delta_{h}^{n}[i]
$$

where

$$
\left(\delta_{h}^{n}[i]\right)(\varphi)= \begin{cases}\alpha_{l} \circ\left(\operatorname{Id}_{A} \otimes \varphi\right) & \text { if } i=0, \\ \varphi \circ\left(\operatorname{Id}_{A \otimes(i-1)} \otimes \mu_{A} \otimes \operatorname{Id}_{A \otimes(n-i)}\right) & \text { if } 1 \leqslant i \leqslant n, \\ \alpha_{r} \circ\left(\varphi \otimes \operatorname{Id}_{A}\right) & \text { if } i=n+1\end{cases}
$$

for $\varphi \in \operatorname{Hoch}^{n}(A, M)$. Here $\alpha_{l}$ and $\alpha_{r}$ are the left and right actions of $A$ on $M$, respectively. The $n$th cohomology module of $\operatorname{Hoch}^{*}(A, M)$ is denoted by $H H^{n}(A, M)$.

\subsection{DGA and Hochschild cup product}

A differential graded associative algebra (DGA in what follows)

$$
C=\left(C^{*}, d^{*}, \cup\right)
$$

consists of a cochain complex $\left(C^{*}, d^{*}\right)$ and an associative graded product on $C^{*}$ such that the Leibniz identity,

$$
d(x \cup y)=(d x) \cup y+(-1)^{|x|} x \cup(d y),
$$

is satisfied for $x, y \in C^{*}$.

Suppose that $M$ is an $A$-bimodule and is an associative algebra itself. Suppose in addition that the following three conditions are satisfied for all $a \in A$ and $m_{1}, m_{2} \in$ $M$ :

$$
\begin{aligned}
& a\left(m_{1} m_{2}\right)=\left(a m_{1}\right) m_{2}, \\
& \left(m_{1} a\right) m_{2}=m_{1}\left(a m_{2}\right), \\
& \left(m_{1} m_{2}\right) a=m_{1}\left(m_{2} a\right) .
\end{aligned}
$$

Then it can be checked easily that the Hochschild cochain complex $\operatorname{Hoch}^{*}(A, M)$ becomes a DGA whose product, denoted by $U$, is given by

$$
(\varphi \cup \psi)\left(a_{1, r+s}\right)=\varphi\left(a_{1, r}\right) \cdot \psi\left(a_{r+1, r+s}\right)
$$

for $\varphi \in \operatorname{Hoch}^{r}(A, M), \psi \in \operatorname{Hoch}^{s}(A, M)$, and $a_{1}, \ldots, a_{r+s} \in A$. Here $a_{p, q}$ denotes the element

$$
a_{p} \otimes \cdots \otimes a_{q}
$$

whenever $p \leqslant q$. We will continue to use this shorthand throughout the rest of this paper.

The three conditions in (3) are satisfied, for example, when $f: A \rightarrow M$ is a morphism of algebras and $A$ acts on $M$ via $f$. 


\section{Deformation bicomplex of module algebras}

From here on until the end of Section $5, A$ will denote an $H$-module algebra with $H$-module structure map $\lambda \in \operatorname{Hom}(H, \operatorname{End}(A))$. The purpose of this section is to construct the deformation bicomplex $C_{\mathrm{MA}}^{* *}(A)$ of $A$. Deformations, which will be discussed in Section 5, are taken with respect to both the $H$-module structure $\lambda$ and the multiplication structure $\mu_{A}$ on $A$. As will be seen below, this bicomplex can be more explicitly denoted by

$$
\operatorname{Hoch}^{*}\left(H, \operatorname{Hom}\left(A^{\otimes *}, A\right)\right) .
$$

Further structures of the deformation bicomplex will be discussed in Section 4 .

\section{1. $H$-bimodule structure on $\operatorname{Hom}\left(A^{\otimes n}, A\right)$}

When $A$ is an $H$-module algebra, there is an $H$-bimodule structure on the module $\operatorname{Hom}\left(A^{\otimes n}, A\right)$ for each $n \geqslant 1$. The left and right actions are given by

$$
\begin{aligned}
& (x \varphi)\left(a_{1, n}\right)=\lambda(x)\left(\varphi\left(a_{1, n}\right)\right), \\
& (\varphi x)\left(a_{1, n}\right)=\sum_{(x)} \varphi\left(\lambda\left(x_{(1)}\right)\left(a_{1}\right) \otimes \cdots \otimes \lambda\left(x_{(n)}\right)\left(a_{n}\right)\right)
\end{aligned}
$$

for $x \in H, \varphi \in \operatorname{Hom}\left(A^{\otimes n}, A\right)$, and $a_{1}, \ldots, a_{n} \in A$.

In particular, there is a Hochschild cochain complex $\operatorname{Hoch}^{*}\left(H, \operatorname{Hom}\left(A^{\otimes n}, A\right)\right)$ of $H$ with coefficients in the $H$-bimodule $\operatorname{Hom}\left(A^{\otimes n}, A\right)$.

\subsection{Deformation bicomplex}

The deformation bicomplex of the $H$-module algebra $A$ is the first quadrant, cohomological bicomplex

$$
C_{\mathrm{MA}}^{* *}(A)=\left\{C_{\mathrm{MA}}^{p, q}(A), d^{p, q},(-1)^{p+1} b^{p, q}\right\}
$$

whose $(p, q)$-entry is

$$
C_{\mathrm{MA}}^{p, q}(A)= \begin{cases}0 & \text { if } p=0, \\ \operatorname{Der}(A) & \text { if }(p, q)=(1,0), \\ \operatorname{Hom}\left(H^{\otimes q}, \operatorname{Hom}\left(A^{\otimes p}, A\right)\right) & \text { otherwise. }\end{cases}
$$

The vertical and horizontal differentials in $C_{\mathrm{MA}}^{* *}(A)$ are denoted by

$$
\begin{aligned}
(-1)^{p+1} b^{p, q}: C_{\mathrm{MA}}^{p, q}(A) & \rightarrow C_{\mathrm{MA}}^{p, q+1}(A), \\
d^{p, q}: C_{\mathrm{MA}}^{p, q}(A) & \rightarrow C_{\mathrm{MA}}^{p+1, q}(A),
\end{aligned}
$$

respectively. These differentials are defined as follows. Note that

$$
C_{\mathrm{MA}}^{p, q}(A)=\operatorname{Hoch}^{q}\left(H, \operatorname{Hom}\left(A^{\otimes p}, A\right)\right) .
$$

For $p \geqslant 1$, set

$$
b^{p, q} \stackrel{\text { def }}{=} \delta_{h}^{q}: \operatorname{Hoch}^{q}\left(H, \operatorname{Hom}\left(A^{\otimes p}, A\right)\right) \rightarrow \operatorname{Hoch}^{q+1}\left(H, \operatorname{Hom}\left(A^{\otimes p}, A\right)\right),
$$


i.e. the Hochschild coboundary. In particular, we have that

$$
b^{p, q}=\sum_{j=0}^{q+1}(-1)^{j} b^{p, q}[j],
$$

where

$$
b^{p, q}[j]=\delta_{h}^{q}[j]
$$

as in (2). The only exception is that $b^{1,0}$ is the restriction of $\delta_{h}^{0}$ to the submodule $\operatorname{Der}(A) \subseteq \operatorname{Hom}(A, A)$. In what follows, such exceptions will be made for the $(1,0)$ entry automatically without further comments.

To define the horizontal differentials, note that

$$
C_{\mathrm{MA}}^{p, 0}(A)=\operatorname{Hom}\left(A^{\otimes p}, A\right)=\operatorname{Hoch}^{p}(A, A) .
$$

In the 0 th row $C_{\mathrm{MA}}^{*, 0}(A)$, the differentials are defined by

$$
d^{p, 0} \stackrel{\text { def }}{=} \delta_{h}^{p}: \operatorname{Hoch}^{p}(A, A) \rightarrow \operatorname{Hoch}^{p+1}(A, A) .
$$

In particular, we have that

$$
d^{p, 0}=\sum_{i=0}^{p+1}(-1)^{i} d^{p, 0}[i]
$$

where

$$
d^{p, 0}[i]=\delta_{h}^{p}[i]
$$

as in (2).

For $q \geqslant 1$, define the horizontal differential by

$$
d^{p, q} \stackrel{\text { def }}{=} \sum_{i=0}^{p+1}(-1)^{i} d^{p, q}[i],
$$

where

$$
\begin{aligned}
& \left(d^{p, q}[i] \varphi\right)\left(x_{1, q}\right)\left(a_{1, p+1}\right)= \\
& \begin{cases}\sum \lambda\left(x_{1(1)} \cdots x_{q(1)}\right)\left(a_{1}\right) \cdot \varphi\left(x_{1(2)} \otimes \cdots \otimes x_{q(2)}\right)\left(a_{2, p+1}\right) & \text { if } i=0 \\
\varphi\left(x_{1, q}\right)\left(a_{1, i-1} \otimes\left(a_{i} a_{i+1}\right) \otimes a_{i+2, p+1}\right) & \text { if } 1 \leqslant i \leqslant p \\
\sum \varphi\left(x_{1(1)} \otimes \cdots \otimes x_{q(1)}\right)\left(a_{1, p}\right) \cdot \lambda\left(x_{1(2)} \cdots x_{q(2)}\right)\left(a_{p+1}\right) & \text { if } i=p+1 .\end{cases}
\end{aligned}
$$

Here $\varphi \in C_{\mathrm{MA}}^{p, q}(A), x_{1}, \ldots, x_{q} \in H$, and $a_{1}, \ldots, a_{p+1} \in A$.

So far we only know that the columns and the 0th row in $C_{\mathrm{MA}}^{* *}(A)$ are cochain complexes. To see that $C_{\mathrm{MA}}^{* *}(A)$ is a bicomplex, we need the following observations.

Theorem 3.1. 1. For each $q \geqslant 1$ and $0 \leqslant k<l \leqslant p+2$, the equality

$$
d^{p+1, q}[l] \circ d^{p, q}[k]=d^{p+1, q}[k] \circ d^{p, q}[l-1]
$$

holds. 
2. For $p \geqslant 1, q \geqslant 0,0 \leqslant i \leqslant p+1$, and $0 \leqslant j \leqslant q+1$, the equality

$$
d^{p, q+1}[i] \circ b^{p, q}[j]=b^{p+1, q}[j] \circ d^{p, q}[i]
$$

holds.

Proof. Both statements can be proved by direct inspections on a case-by-case basis. For instance, for (6) when $i=j=0$, we have that

$$
\begin{aligned}
& \left(b^{p+1, q}[0] \circ d^{p, q}[0]\right)(\varphi)\left(x_{1, q+1}\right)\left(a_{1, p+1}\right) \\
& =\lambda\left(x_{1}\right)\left(\left(d^{p, q}[0]\right)(\varphi)\left(x_{2, q+1}\right)\left(a_{1, p+1}\right)\right) \\
& =\lambda\left(x_{1}\right)\left(\sum \lambda\left(x_{2(1)} \cdots x_{q+1(1)}\right)\left(a_{1}\right) \cdot \varphi\left(x_{2(2)} \otimes \cdots \otimes x_{q+1(2)}\right)\left(a_{2, p+1}\right)\right) \\
& \stackrel{(i)}{=} \sum \lambda\left(x_{1(1)}\right)\left(\lambda\left(x_{2(1)} \cdots x_{q+1(1)}\right)\left(a_{1}\right)\right) . \\
& \quad \lambda\left(x_{1(2)}\right)\left(\varphi\left(x_{2(2)} \otimes \cdots \otimes x_{q+1(2)}\right)\left(a_{2, p+1}\right)\right) \\
& \stackrel{(i i)}{=} \sum \lambda\left(x_{1(1)} \cdots x_{q+1(1)}\right)\left(a_{1}\right) \cdot \lambda\left(x_{1(2)}\right)\left(\varphi\left(x_{2(2)} \otimes \cdots \otimes x_{q+1(2)}\right)\left(a_{2, p+1}\right)\right) \\
& =\sum \lambda\left(x_{1(1)} \cdots x_{q+1(1)}\right)\left(a_{1}\right) \cdot\left(b^{p, q}[0] \varphi\right)\left(x_{1(2)} \otimes \cdots \otimes x_{q+1(2)}\right)\left(a_{2, p+1}\right) \\
& =\left(d^{p, q+1}[0] \circ b^{p, q}[0]\right)(\varphi)\left(x_{1, q+1}\right)\left(a_{1, p+1}\right) .
\end{aligned}
$$

Here the equalities (i) and (ii) follow from the module algebra axioms (1).

Similarly, if $i=0$ and $1 \leqslant j \leqslant q$, then we have that

$$
\begin{aligned}
& \left(b^{p+1, q}[j] \circ d^{p, q}[0]\right)(\varphi)\left(x_{1, q+1}\right)\left(a_{1, p+1}\right) \\
& =\sum \lambda\left(x_{1(1)} \cdots x_{q+1(1)}\right)\left(a_{1}\right) . \\
& \quad \varphi\left(x_{1(2)} \otimes \cdots \otimes x_{j-1(2)} \otimes\left(x_{j(2)} x_{j+1(2)}\right) \otimes x_{j+2(2)} \otimes \cdots \otimes x_{q+1(2)}\right)\left(a_{2, p+1}\right) \\
& =\left(d^{p, q+1}[0] \circ b^{p, q}[j]\right)(\varphi)\left(x_{1, q+1}\right)\left(a_{1, p+1}\right) .
\end{aligned}
$$

Note that only the bialgebra structure on $H$ is used in proving this condition.

Likewise, when $i=0$ and $j=q+1$, we have that

$$
\begin{aligned}
& \left(b^{p+1, q}[q+1] \circ d^{p, q}[0]\right)(\varphi)\left(x_{1, q+1}\right)\left(a_{1, p+1}\right) \\
& =\sum \lambda\left(x_{1(1)} \cdots x_{q+1(1)}\right)\left(a_{1}\right) . \\
& \quad \varphi\left(x_{1(2)} \otimes \cdots \otimes x_{q(2)}\right)\left(\lambda\left(x_{q+1(2)}\right)\left(a_{2}\right) \otimes \cdots \otimes \lambda\left(x_{q+1(p+1)}\right)\left(a_{p+1}\right)\right) \\
& =\left(d^{p, q+1}[0] \circ b^{p, q}[q+1]\right)(\varphi)\left(x_{1, q+1}\right)\left(a_{1, p+1}\right) .
\end{aligned}
$$

This proves (6) when $i=0$.

If $1 \leqslant i \leqslant p$, then we have that

$$
\begin{aligned}
& \left(d^{p, q+1}[i] \circ b^{p, q}[j]\right)(\varphi)\left(x_{1, q+1}\right)\left(a_{1, p+1}\right)= \\
& \begin{cases}\lambda\left(x_{1}\right)\left(\varphi\left(x_{2, q+1}\right)\left(a_{1, i-1} \otimes\left(a_{i} a_{i+1}\right) \otimes a_{i+2, p+1}\right)\right) & \text { if } j=0, \\
\varphi\left(x_{1, j-1} \otimes\left(x_{j} x_{j+1}\right) \otimes x_{j+2, q+1}\right)\left(a_{1, i-1} \otimes\left(a_{i} a_{i+1}\right) \otimes a_{i+2, p+1}\right) & \text { if } 1 \leqslant j \leqslant q, \\
\sum \varphi\left(x_{1, q}\right)\left(\lambda\left(x_{q+1(1)}\right)\left(a_{1}\right) \otimes \cdots\right. & \text { if } j=q+1 \\
\left.\otimes \lambda\left(x_{q+1(i)}\right)\left(a_{i} a_{i+1}\right) \otimes \cdots \otimes \lambda\left(x_{q+1(p)}\right)\left(a_{p+1}\right)\right) & \end{cases} \\
& =\left(b^{p+1, q}[j] \circ d^{p, q}[i]\right)(\varphi)\left(x_{1, q+1}\right)\left(a_{1, p+1}\right) .
\end{aligned}
$$


This proves $(6)$ when $1 \leqslant i \leqslant p$. This cases when $i=p+1$ are similar to the cases when $i=0$.

The condition (5) is proved by a similar analysis.

From the condition (5), it follows as usual that

$$
d^{p+1, q} \circ d^{p, q}=0 .
$$

Therefore, each row $\left(C_{\mathrm{MA}}^{*, q}(A), d^{*, q}\right)$ is a cochain complex. From the condition (6), it follows that

$$
d^{p, q+1} \circ b^{p, q}=b^{p+1, q} \circ d^{p, q} .
$$

In other words, each square in $C_{\mathrm{MA}}^{* *}(A)$ anti-commutes. This leads to the following result.

Corollary 3.2. The above definitions give a first quadrant, cohomological bicomplex $C_{\mathrm{MA}}^{* *}(A)=\left\{C_{\mathrm{MA}}^{p, q}, d^{p, q},(-1)^{p+1} b^{p, q}\right\}$.

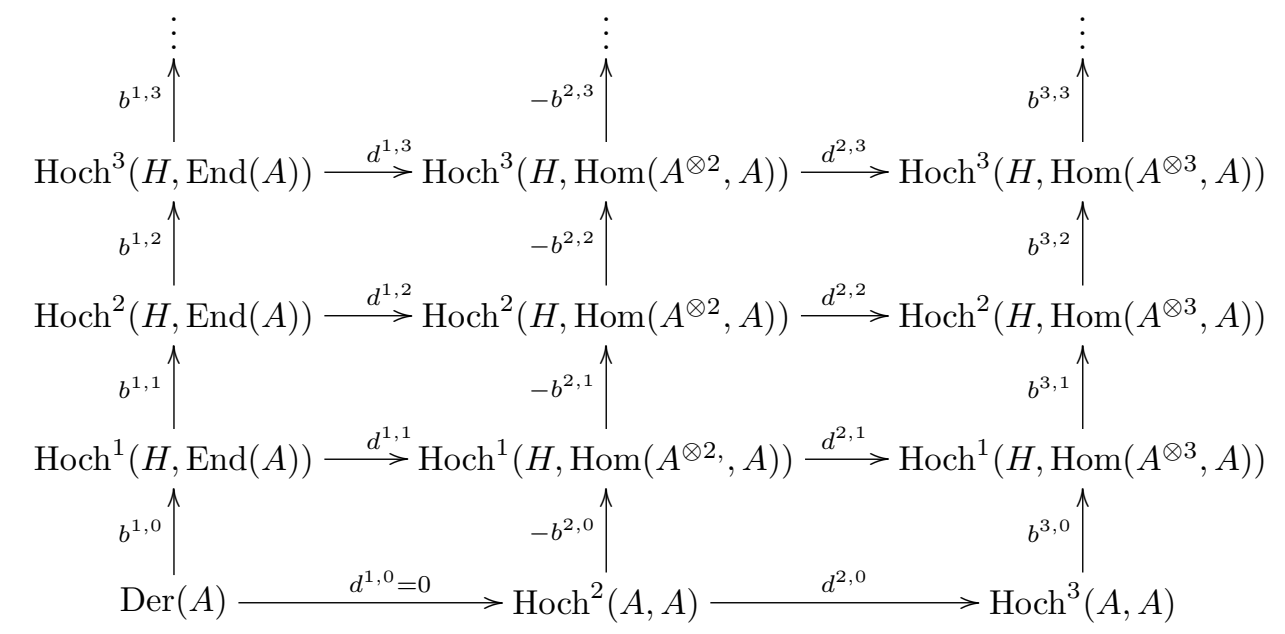

The bicomplex $C_{\mathrm{MA}}^{* *}(A)$ is called the deformation bicomplex of $A$. Omitting the 0th column, which is the 0 cochain complex, the first three columns of $C_{\mathrm{MA}}^{* *}(A)$ appear as above.

Note that the deformation bicomplex $C_{\mathrm{MA}}^{* *}(A)$ contains the cochain complex $\mathcal{F}^{*}(A)$ constructed in [20]. In fact, $\mathcal{F}^{*}(A)$ is, up to a shift in degree, the total complex of the sub-bicomplex of $C_{\mathrm{MA}}^{* *}(A)$ consisting of the first row $C_{\mathrm{MA}}^{*, 1}(A)$ and the first column $C_{\mathrm{MA}}^{1, *}(A)$.

\subsection{Total complex and cohomology}

Denote by $\left(C_{\mathrm{MA}}^{*}(A), d_{\mathrm{MA}}^{*}\right)$ the total complex of the deformation bicomplex $C_{\mathrm{MA}}^{* *}(A)$. In particular:

$$
C_{\mathrm{MA}}^{n}(A)= \begin{cases}0 & \text { if } n=0 \\ \operatorname{Der}(A) & \text { if } n=1 \\ \bigoplus_{i=1}^{n} \operatorname{Hoch}^{n-i}\left(H, \operatorname{Hom}\left(A^{\otimes i}, A\right)\right) & \text { if } n \geqslant 2\end{cases}
$$


The cochain complex $\left(C_{\mathrm{MA}}^{*}(A), d_{\mathrm{MA}}^{*}\right)$ is called the deformation complex of $A$.

Define the cohomology module

$$
H_{\mathrm{MA}}^{n}(A) \stackrel{\text { def }}{=} H^{n}\left(C_{\mathrm{MA}}^{*}(A), d_{\mathrm{MA}}^{*}\right) .
$$

The deformation complex and its cohomology modules will be used in Section 5 to study algebraic deformations of $A$.

\section{Cup product}

In this section, we observe that each row in the deformation bicomplex $C_{\mathrm{MA}}^{* *}(A)$ is a differential graded associative algebra. Moreover, each row $C_{\mathrm{MA}}^{*, q}(A)$ is canonically isomorphic to a Hochschild cochain complex. Under this isomorphism, the product in each row corresponds to a Hochschild $\cup$-product.

\subsection{Cup product in $C_{\mathrm{MA}}^{*, q}(A)$}

The Hochschild $\cup$-product in the 0th row

$$
C_{\mathrm{MA}}^{*, 0}(A)=\operatorname{Hoch}^{*}(A, A)
$$

generalizes to the higher rows $C_{\mathrm{MA}}^{*, q}(A)(q \geqslant 1)$. In fact, one can define a product

$$
\begin{aligned}
-\cup-: \operatorname{Hom}\left(H^{\otimes q}, \operatorname{Hom}\left(A^{\otimes r}, A\right)\right) \otimes \operatorname{Hom}\left(H^{\otimes q},\right. & \left.\operatorname{Hom}\left(A^{\otimes s}, A\right)\right) \\
& \rightarrow \operatorname{Hom}\left(H^{\otimes q}, \operatorname{Hom}\left(A^{\otimes(r+s)}, A\right)\right)
\end{aligned}
$$

by setting

$$
\begin{aligned}
& (\varphi \cup \psi)\left(x_{1, q}\right)\left(a_{1, r+s}\right) \\
& \quad \stackrel{\text { def }}{=} \sum \varphi\left(x_{1(1)} \otimes \cdots \otimes x_{q(1)}\right)\left(a_{1, r}\right) \cdot \psi\left(x_{1(2)} \otimes \cdots \otimes x_{q(2)}\right)\left(a_{r+1, r+s}\right)
\end{aligned}
$$

for $\varphi \in \operatorname{Hom}\left(H^{\otimes q}, \operatorname{Hom}\left(A^{\otimes r}, A\right)\right), \psi \in \operatorname{Hom}\left(H^{\otimes q}, \operatorname{Hom}\left(A^{\otimes s}, A\right)\right), x_{i} \in H$, and $a_{j} \in$ A.

Theorem 4.1. The product defined in (7) is associative and satisfies the Leibniz identity,

$$
d^{r+s, q}(\varphi \cup \psi)=\left(d^{r, q} \varphi\right) \cup \psi+(-1)^{r} \varphi \cup\left(d^{s, q} \psi\right) .
$$

In particular, $\left(C_{\mathrm{MA}}^{*, q}(A), d^{*, q}, \cup\right)$ is a $D G A$.

Proof. The associativity of the $\cup$-product is a consequence of the coassociativity of the comultiplication $\Delta_{H}$ in $H$ and the associativity of the multiplication $\mu_{A}$ in $A$. The Leibniz identity can be checked by a simple inspection of both sides of (8) when applied to $x_{1, q} \in H^{\otimes q}$ and then to $a_{1, r+s+1} \in A^{\otimes(r+s+1)}$.

The Leibniz identity implies, as usual, that the $\cup$-product descends to cohomology, which leads to the following consequence. 
Corollary 4.2. For each $q \geqslant 0$,

$$
\left(\bigoplus_{n \geqslant 1} H^{n}\left(C_{\mathrm{MA}}^{*, q}(A), d^{*, q}\right), \cup\right)
$$

is a graded associative algebra.

There is a more conceptual way to obtain the $\cup$-product above by realizing it as a Hochschild U-product, which we do next.

\subsection{Alternative description of $C_{\mathrm{MA}}^{*, q}(A)$}

Fix an integer $q \geqslant 1$. Using the second module algebra axiom in (1), one obtains an $A$-bimodule structure on the module $\operatorname{Hom}\left(H^{\otimes q}, A\right)$ via the actions

$$
\begin{aligned}
& (a f)\left(x_{1, q}\right)=\sum \lambda\left(x_{1(1)} \cdots x_{q(1)}\right)(a) \cdot f\left(x_{1(2)} \otimes \cdots \otimes x_{q(2)}\right), \\
& (f a)\left(x_{1, q}\right)=\sum f\left(x_{1(1)} \otimes \cdots \otimes x_{q(1)}\right) \cdot \lambda\left(x_{1(2)} \cdots x_{q(2)}\right)(a)
\end{aligned}
$$

for $a \in A, f \in \operatorname{Hom}\left(H^{\otimes q}, A\right)$, and $x_{1}, \ldots, x_{q} \in H$.

Moreover, the module $\operatorname{Hom}\left(H^{\otimes q}, A\right)$ is an associative algebra via the product

$$
(f \cdot g)\left(x_{1, q}\right)=\sum f\left(x_{1(1)} \otimes \cdots \otimes x_{q(1)}\right) \cdot g\left(x_{1(2)} \otimes \cdots \otimes x_{q(2)}\right)
$$

for $f, g \in \operatorname{Hom}\left(H^{\otimes q}, A\right)$ and $x_{i} \in H$. The three conditions in (3) can be checked easily in this case. It follows as in Section 2.4 that the Hochschild cochain complex $\operatorname{Hoch}^{*}\left(A, \operatorname{Hom}\left(H^{\otimes q}, A\right)\right)$ is a DGA whose associative $\cup$-product is given by

$$
\begin{aligned}
(\varphi \cup \psi)\left(a_{1, r+s}\right) & \left(x_{1, q}\right) \\
= & \sum \varphi\left(a_{1, r}\right)\left(x_{1(1)} \otimes \cdots \otimes x_{q(1)}\right) \cdot \psi\left(a_{r+1, r+s}\right)\left(x_{1(2)} \otimes \cdots \otimes x_{q(2)}\right)
\end{aligned}
$$

for $\varphi \in \operatorname{Hoch}^{r}\left(A, \operatorname{Hom}\left(H^{\otimes q}, A\right)\right), \psi \in \operatorname{Hoch}^{s}\left(A, \operatorname{Hom}\left(H^{\otimes q}, A\right)\right), a_{i} \in A$, and $x_{j} \in H$.

Theorem 4.3. There is a canonical isomorphism

$$
\zeta:\left(\operatorname{Hoch}^{p}\left(A, \operatorname{Hom}\left(H^{\otimes q}, A\right)\right), \delta_{h}^{p}, \cup\right)_{p \geqslant 1} \stackrel{\cong}{\rightrightarrows}\left(C_{\mathrm{MA}}^{*, q}(A), d^{*, q}, \cup\right)
$$

of DGAs defined by

$$
(\zeta \varphi)\left(x_{1, q}\right)\left(a_{1, p}\right) \stackrel{\text { def }}{=} \varphi\left(a_{1, p}\right)\left(x_{1, q}\right)
$$

for $\varphi \in \operatorname{Hoch}^{p}\left(A, \operatorname{Hom}\left(H^{\otimes q}, A\right)\right), x_{i} \in H$, and $a_{j} \in A$.

Proof. It is clear that the map $\zeta$ defined in (9) is a linear isomorphism. In fact, it is just the usual Hom $-\otimes$ adjunction applied twice. Direct inspections then show that under the isomorphism $\zeta, \delta_{h}^{*}$ corresponds to $d^{*, q}$ and the $\cup$-product in $\operatorname{Hoch}^{*}\left(A, \operatorname{Hom}\left(H^{\otimes q}\right.\right.$, $A)$ ) corresponds to the one in $C_{\mathrm{MA}}^{*, q}(A)$.

Passing to cohomology, this leads to the following result. 
Corollary 4.4. The isomorphism $\zeta$ (9) induces an isomorphism

$$
\zeta:\left(\bigoplus_{n \geqslant 1} H H^{n}\left(A, \operatorname{Hom}\left(H^{\otimes q}, A\right)\right), \cup\right) \cong\left(\bigoplus_{n \geqslant 1} H^{n}\left(C_{\mathrm{MA}}^{*, q}(A), d^{*, q}\right), \cup\right)
$$

of graded associative algebras.

Note that the $\cup$-product in the first row $C_{\mathrm{MA}}^{*, 1}(A)$ coincides with the one in $\mathcal{F}_{1}^{*}(A)$ constructed in $[\mathbf{2 0}]$.

\section{Deformations of module algebras}

In this section, we show that the deformation complex $C_{\mathrm{MA}}^{*}(A)$ is actually the cochain complex that controls the deformations, in the sense of Gerstenhaber [6], of an $H$-module algebra $A$, in which both the $H$-module structure $\lambda \in \operatorname{Hom}(H, \operatorname{End}(A))$ and the multiplication $\mu_{A} \in \operatorname{Hom}\left(A^{\otimes 2}, A\right)$ on $A$ are deformed. This generalizes the treatment in [20], in which only the $H$-module structure is deformed. However, once the correct definitions are made, the arguments here are similar to those in $[\mathbf{2 0}]$.

\subsection{Deformation}

A deformation of $A$ is a power series

$$
\Theta=\sum_{n \geqslant 0} \theta_{n} t^{n}
$$

with $\theta_{0}=\left(\lambda, \mu_{A}\right) \in C_{\mathrm{MA}}^{2}(A)$ and each $\theta_{n}=\left(\lambda_{n}, \pi_{n}\right) \in C_{\mathrm{MA}}^{2}(A)$, satisfying the following three conditions. Writing

$$
\Lambda=\sum_{n \geqslant 0} \lambda_{n} t^{n} \quad\left(\lambda_{0}=\lambda\right)
$$

and

$$
\Pi=\sum_{n \geqslant 0} \pi_{n} t^{n} \quad\left(\pi_{0}=\mu_{A}\right)
$$

the three conditions are:

$$
\begin{aligned}
\Lambda(x y) & =\Lambda(x) \circ \Lambda(y), \\
\Lambda(x)(\Pi(a, b)) & =\sum_{(x)} \Pi\left(\Lambda\left(x_{(1)}\right)(a), \Lambda\left(x_{(2)}\right)(b)\right), \\
\Pi(\Pi(a, b), c) & =\Pi(a, \Pi(b, c))
\end{aligned}
$$

for $x, y \in H$ and $a, b, c \in A$. Such a deformation will also be denoted by $\Theta=(\Lambda, \Pi)$.

The linear coefficient $\theta_{1}=\left(\lambda_{1}, \pi_{1}\right)$ of a deformation $\Theta$ is called the infinitesimal. The trivial deformation is the deformation $\Theta=\theta_{0}=\left(\lambda, \mu_{A}\right)$. 


\subsection{Equivalence}

A formal automorphism of $A$ is a power series

$$
\Phi=\sum_{n \geqslant 0} \phi_{n} t^{n},
$$

in which $\phi_{0}=\operatorname{Id}_{A}$ and each $\phi_{n} \in \operatorname{End}(A)$, such that the first non-zero $\phi_{n}(n \geqslant 1)$ is a derivation on $A$. Note that $\phi_{1}$ is necessarily a derivation on $A$.

Two deformations $\Theta=(\Lambda, \Pi)$ and $\bar{\Theta}=(\bar{\Lambda}, \bar{\Pi})$ are said to be equivalent if and only if there exists a formal automorphism $\Phi$ such that the following two conditions are satisfied:

$$
\begin{aligned}
& \bar{\Pi}=\Phi^{-1} \circ \Pi \circ \Phi^{\otimes 2}, \\
& \bar{\Lambda}=\Phi^{-1} \Lambda \Phi .
\end{aligned}
$$

On the right-hand side of (11b), we use the interpretation

$$
\left(\phi_{i} \lambda_{j} \phi_{k}\right)(x)=\phi_{i} \circ \lambda_{j}(x) \circ \phi_{k}
$$

for $x \in H$. In the above situation, we write

$$
\bar{\Theta}=\Phi^{-1} \Theta \Phi .
$$

This defines an equivalence relation.

Given a deformation $\Theta=(\Lambda, \Pi)$ and a formal automorphism $\Phi$, one can define an equivalent deformation $\bar{\Theta}=\Phi^{-1} \Theta \Phi$ using (11a) and (11b).

The $H$-module algebra $A$ is said to be rigid if every deformation of $A$ is equivalent to the trivial deformation.

Theorem 5.1. Let $A$ be an $H$-module algebra. Then the following statements hold.

1. The infinitesimal $\theta_{1}$ of any deformation $\Theta$ of $A$ is a 2-cocycle in the deformation complex $C_{\mathrm{MA}}^{2}(A)$ whose cohomology class is determined by the equivalence class of $\Theta$.

2. If $H_{\mathrm{MA}}^{2}(A)=0$, then $A$ is rigid.

Proof. The deformation arguments in [6] and [20] can be adapted to the present situation. For example, the deformation axioms (10) can be rewritten as:

$$
\begin{aligned}
\lambda_{n}(x y) & =\sum_{i+j=n} \lambda_{i}(x) \circ \lambda_{j}(y), \\
\sum_{i+j=n} \lambda_{i}(x)\left(\pi_{j}(a, b)\right) & =\sum_{(x)} \sum_{i+j+k=n} \pi_{i}\left(\lambda_{j}\left(x_{(1)}\right)(a), \lambda_{k}\left(x_{(2)}\right)(b)\right), \\
\sum_{i+j=n} \pi_{i}\left(\pi_{j}(a, b), c\right) & =\sum_{i+j=n} \pi_{i}\left(a, \pi_{j}(b, c)\right)
\end{aligned}
$$

for $n \geqslant 1, x, y \in H$, and $a, b, c \in A$. When $n=1$, these three conditions state that

$$
\begin{aligned}
b^{1,1} \lambda_{1} & =0, \\
d^{1,1} \lambda_{1}-b^{2,0} \pi_{1} & =0, \\
d^{2,0} \pi_{1} & =0 .
\end{aligned}
$$

These three statements together state that $\theta_{1}=\left(\lambda_{1}, \pi_{1}\right) \in C_{\mathrm{MA}}^{2}(A)$ is a 2-cocycle. 
Moreover, if $\bar{\Theta}=\Phi^{-1} \Theta \Phi$ for some formal automorphism $\Phi$, then the condition on the linear coefficients can be restated as

$$
\bar{\theta}_{1}-\theta_{1}=d_{\mathrm{MA}}^{1} \phi_{1},
$$

which is a 2-coboundary in $C_{\mathrm{MA}}^{2}(A)$. This proves statement (1). Statement (2) is proved similarly by adapting Proposition 3.5 in $[\mathbf{2 0}]$.

\section{Deformation bicomplex of module coalgebras}

In this section, the deformation bicomplex

$$
C_{\mathrm{MC}}^{* *}(A)=\operatorname{Hoch}^{*}\left(H, \operatorname{Hom}\left(A, A^{\otimes *}\right)\right)
$$

for an $H$-module coalgebra $A$ is constructed, in which both the $H$-module structure and the coalgebra structure on $A$ are deformed. The corresponding deformation results are then listed. The proofs can be adapted from the module algebra case.

\subsection{Module coalgebra}

Throughout this section, let $A=\left(A, \Delta_{A}\right)$ be a coassociative coalgebra. A coderivation on $A$ is a linear self-map $\varphi \in \operatorname{Hom}(A, A)$ such that

$$
\Delta_{A} \circ \varphi=\left(\operatorname{Id}_{A} \otimes \varphi+\varphi \otimes \operatorname{Id}_{A}\right) \circ \Delta_{A} .
$$

The set of coderivations on $A$ is denoted by $\operatorname{Coder}(A)$, which is considered as a submodule of $\operatorname{Hom}(A, A)$.

An $H$-module coalgebra structure on $A$ consists of an $H$-module structure $\lambda \in$ $\operatorname{Hom}(H, \operatorname{End}(A))$ on $A$ such that the map $\Delta_{A}: A \rightarrow A \otimes A$ becomes an $H$-module morphism, i.e.,

$$
\Delta_{A}(\lambda(x)(a))=\sum_{(a)(x)} \lambda\left(x_{(1)}\right)\left(a_{(1)}\right) \otimes \lambda\left(x_{(2)}\right)\left(a_{(2)}\right)
$$

for all $x \in H$ and $a \in A$. For the rest of this section, $A$ will denote an $H$-module coalgebra with $H$-module structure map $\lambda$.

\subsection{Hochschild coalgebra cohomology}

The deformation bicomplex of $A$ uses the coalgebra version of Hochschild cohomology $[\mathbf{1 0}, \mathbf{1 7}]$, which we now recall.

Let $M$ be an $A$-bicomodule with left $A$-coaction $\psi_{l}$ and right $A$-coaction $\psi_{r}$. Define the module of Hochschild coalgebra n-cochains of $A$ with coefficients in $M$ as

$$
\operatorname{Hoch}_{c}^{n}(M, A)= \begin{cases}0 & \text { if } n=0, \\ \operatorname{Hom}\left(M, A^{\otimes n}\right) & \text { if } n \geqslant 1 .\end{cases}
$$

The coboundary is defined by

$$
\begin{aligned}
\delta_{c} \sigma=\left(\operatorname{Id}_{A} \otimes \sigma\right) \circ \psi_{l}+\sum_{i=1}^{n}(-1)^{i}\left(\operatorname{Id}_{A \otimes(i-1)} \otimes \Delta_{A} \otimes \operatorname{Id}_{A \otimes(n-i)}\right) \circ \sigma & \\
& +(-1)^{n+1}\left(\sigma \otimes \operatorname{Id}_{A}\right) \circ \psi_{r}
\end{aligned}
$$

for $\sigma \in \operatorname{Hoch}_{c}^{n}(M, A)$. 
Suppose, in addition, that $M=\left(M, \Delta_{M}\right)$ is a coassociative coalgebra such that the following three conditions, dual to (3), are satisfied:

$$
\begin{aligned}
\left(\operatorname{Id}_{A} \otimes \Delta_{M}\right) \circ \psi_{l} & =\left(\psi_{l} \otimes \operatorname{Id}_{M}\right) \circ \Delta_{M}, \\
\left(\Delta_{M} \otimes \operatorname{Id}_{A}\right) \circ \psi_{r} & =\left(\operatorname{Id}_{M} \otimes \psi_{r}\right) \circ \Delta_{M}, \\
\left(\psi_{r} \otimes \operatorname{Id}_{M}\right) \circ \Delta_{M} & =\left(\operatorname{Id}_{M} \otimes \psi_{l}\right) \circ \Delta_{M} .
\end{aligned}
$$

Then the Hochschild coalgebra cochain $\operatorname{complex}_{\operatorname{Hoch}_{c}^{*}}(M, A)$ becomes a DGA with the product

$$
f \cup g=(f \otimes g) \circ \Delta_{M}
$$

for $f, g \in \operatorname{Hoch}_{c}^{*}(M, A)$.

\section{3. $\quad H$-bimodule structure on $\operatorname{Hom}\left(A, A^{\otimes n}\right)$}

For $n \geqslant 1$, there is an $H$-bimodule structure on $\operatorname{Hom}\left(A, A^{\otimes n}\right)$ defined as follows. The left and right $H$-actions on $\operatorname{Hom}\left(A, A^{\otimes n}\right)$ are given by

$$
\begin{aligned}
& (x \varphi)(a)=\sum \lambda\left(x_{(1)}\right)\left(\varphi(a)^{1}\right) \otimes \cdots \otimes \lambda\left(x_{(n)}\right)\left(\varphi(a)^{n}\right), \\
& (\varphi x)(a)=\varphi(\lambda(x)(a))
\end{aligned}
$$

for $x \in H, \varphi \in \operatorname{Hom}\left(A, A^{\otimes n}\right)$, and $a \in A$. In the left $H$-action, we use the notation

$$
\varphi(a)=\sum \varphi(a)^{1} \otimes \cdots \otimes \varphi(a)^{n} \in A^{\otimes n} .
$$

In particular, we can consider the Hochschild cochain complex

$$
\operatorname{Hoch}^{*}\left(H, \operatorname{Hom}\left(A, A^{\otimes n}\right)\right) \text {. }
$$

\subsection{Module coalgebra deformation bicomplex}

The deformation bicomplex of the $H$-module coalgebra $A$ is the first quadrant, cohomological bicomplex

$$
C_{\mathrm{MC}}^{* *}(A)=\left\{C_{\mathrm{MC}}^{p, q}(A), d^{p, q},(-1)^{p+1} b^{p, q}\right\}
$$

with

$$
C_{\mathrm{MC}}^{p, q}(A)= \begin{cases}0 & \text { if } p=0, \\ \operatorname{Coder}(A) & \text { if }(p, q)=(1,0), \\ \operatorname{Hom}\left(H^{\otimes q}, \operatorname{Hom}\left(A, A^{\otimes p}\right)\right) & \text { otherwise. }\end{cases}
$$

For each $p \geqslant 1$, set

$$
b^{p, q} \stackrel{\text { def }}{=} \delta_{h}^{q}: \operatorname{Hoch}^{q}\left(H, \operatorname{Hom}\left(A, A^{\otimes p}\right)\right) \rightarrow \operatorname{Hoch}^{q+1}\left(H, \operatorname{Hom}\left(A, A^{\otimes p}\right)\right) .
$$

In the 0th row $C_{\mathrm{MC}}^{*, 0}(A)$, define the horizontal differential as the Hochschild coalgebra coboundary,

$$
d^{*, 0} \stackrel{\text { def }}{=} \delta_{c}^{*}: \operatorname{Hoch}_{c}^{*}(A, A) \rightarrow \operatorname{Hoch}_{c}^{*+1}(A, A) .
$$

For $q \geqslant 1$, the horizontal differential

$$
d^{p, q}: C_{\mathrm{MC}}^{p, q}(A) \rightarrow C_{\mathrm{MC}}^{p+1, q}(A)
$$


is defined as the alternating sum

$$
d^{p, q}=\sum_{i=0}^{p+1}(-1)^{i} d^{p, q}[i]
$$

where

$$
\begin{aligned}
& \left(d^{p, q}[i] \varphi\right)\left(x_{1, q}\right)(a)= \\
& \begin{cases}\sum \lambda\left(x_{1(1)} \cdots x_{q(1)}\right)\left(a_{(1)}\right) \otimes \varphi\left(x_{1(2)} \otimes \cdots \otimes x_{q(2)}\right)\left(a_{(2)}\right) & \text { if } i=0, \\
\left(\operatorname{Id}_{A \otimes(i-1)} \otimes \Delta_{A} \otimes \operatorname{Id}_{A \otimes(p-i)}\right)\left(\varphi\left(x_{1, q}\right)(a)\right) & \text { if } 1 \leqslant i \leqslant p, \\
\sum \varphi\left(x_{1(1)} \otimes \cdots \otimes x_{q(1)}\right)\left(a_{(1)}\right) \otimes \lambda\left(x_{1(2)} \cdots x_{q(2)}\right)\left(a_{(2)}\right) & \text { if } i=p+1 .\end{cases}
\end{aligned}
$$

Here $\varphi \in C_{\mathrm{MC}}^{p, q}(A), x_{j} \in H$, and $a \in A$.

Theorem 3.1 still holds in the module coalgebra case with essentially the same proof. It follows that $C_{\mathrm{MC}}^{* *}(A)$ is indeed a bicomplex. Denote its total complex by $C_{\mathrm{MC}}^{*}(A)$, which is called the deformation complex of $A$. The $n$th cohomology module of $C_{\mathrm{MC}}^{*}(A)$ is denoted by $H_{\mathrm{MC}}^{n}(A)$.

Note that the cochain complex $\mathcal{F}_{m c}^{*}(A)$ constructed in $[20]$ is the total complex of the sub-bicomplex of $C_{\mathrm{MC}}^{* *}(A)$ consisting of the first column $C_{\mathrm{MC}}^{1, * *}(A)$ and the first row $C_{\mathrm{MC}}^{*, 1}(A)$.

\subsection{Cup product}

As in the module algebra case, each row $\left(C_{\mathrm{MC}}^{*, q}(A), d^{*, q}\right)$ is a DGA whose product is defined by

$$
(\varphi \cup \psi)\left(x_{1, q}\right)(a) \stackrel{\text { def }}{=} \sum \varphi\left(x_{1(1)} \otimes \cdots \otimes x_{q(1)}\right)\left(a_{(1)}\right) \otimes \psi\left(x_{1(2)} \otimes \cdots \otimes x_{q(2)}\right)\left(a_{(2)}\right)
$$

for $\varphi \in C_{\mathrm{MC}}^{r, q}(A)$ and $\psi \in C_{\mathrm{MC}}^{s, q}(A)$.

\subsection{Module coalgebra deformation}

A deformation of the $H$-module coalgebra $A$ is a power series $\Theta=\sum_{n \geqslant 0} \theta_{n} t^{n}$ with $\theta_{0}=\left(\lambda, \Delta_{A}\right)$ and each $\theta_{n}=\left(\lambda_{n}, \Delta_{n}\right) \in C_{\mathrm{MC}}^{2}(A)$ such that the following three conditions are satisfied. Writing

$$
\Lambda=\sum_{n \geqslant 0} \lambda_{n} t^{n} \quad\left(\lambda_{0}=\lambda\right)
$$

and

$$
D=\sum_{n \geqslant 0} \Delta_{n} t^{n} \quad\left(\Delta_{0}=\Delta_{A}\right)
$$

the three conditions are:

$$
\begin{aligned}
\Lambda(x y) & =\Lambda(x) \circ \Lambda(y), \\
D \circ \Lambda(x) & =\left\{\sum_{(x)} \Lambda\left(x_{(1)}\right) \otimes \Lambda\left(x_{(2)}\right)\right\} \circ D, \\
\left(D \otimes \operatorname{Id}_{A}\right) \circ D & =\left(\operatorname{Id}_{A} \otimes D\right) \circ D
\end{aligned}
$$

for $x, y \in H$. Such a deformation is also denoted by $\Theta=(\Lambda, D)$. The linear coefficient $\theta_{1} \in C_{\mathrm{MC}}^{2}(A)$ is called the infinitesimal. The trivial deformation is the deformation 
$\Theta=\theta_{0}=\left(\lambda, \Delta_{A}\right)$.

A formal automorphism of $A$ is a power series $\Phi=\sum_{n \geqslant 0} \phi_{n} t^{n}$ with $\phi_{0}=\operatorname{Id}_{A}$ and each $\phi_{n} \in \operatorname{End}(A)$ such that the first non-zero $\phi_{n}(n \geqslant 1)$ is a coderivation on $A$.

Two deformations $\Theta=(\Lambda, D)$ and $\bar{\Theta}=(\bar{\Lambda}, \bar{D})$ are said to be equivalent if and only if there exists a formal automorphism $\Phi$ such that the following two conditions hold:

$$
\begin{aligned}
& \bar{D}=\left(\Phi^{-1}\right)^{\otimes 2} \circ D \circ \Phi, \\
& \bar{\Lambda}=\Phi^{-1} \Lambda \Phi .
\end{aligned}
$$

The $H$-module coalgebra $A$ is said to be rigid if every deformation of $A$ is equivalent to the trivial deformation.

The following result is the module coalgebra analogue of Theorem 5.1 and can be proved by similar arguments.

Theorem 6.1. Let $A$ be an $H$-module coalgebra. Then the following statements hold.

1. The infinitesimal $\theta_{1}$ of any deformation $\Theta$ of $A$ is a 2-cocycle in the deformation complex $C_{\mathrm{MC}}^{2}(A)$ whose cohomology class is determined by the equivalence class of $\Theta$.

2. If $H_{\mathrm{MC}}^{2}(A)=0$, then $A$ is rigid.

\section{Deformation bicomplex of comodule algebras}

The purposes of this section are to construct the deformation bicomplex

$$
C_{\mathrm{CA}}^{* *}(A)=\operatorname{Hoch}^{*}\left(A, H^{\otimes *} \otimes A\right)
$$

of an $H$-comodule algebra $A$ and to list the corresponding deformation results. Deformations are taken with respect to both the $H$-comodule structure and the algebra structure on $A$.

\subsection{Comodule algebra}

For an associative algebra $A=\left(A, \mu_{A}\right)$, an $H$-comodule algebra structure on $A$ consists of an $H$-comodule structure $\rho \in \operatorname{Hom}(A, H \otimes A)$ on $A$ such that the map $\mu_{A}: A \otimes A \rightarrow A$ becomes an $H$-comodule morphism, i.e.,

$$
\rho \circ \mu_{A}=\left(\mu_{H} \otimes \mu_{A}\right) \circ\left(\operatorname{Id}_{H} \otimes \tau_{(A, H)} \otimes \operatorname{Id}_{A}\right) \circ \rho^{\otimes 2} .
$$

Here and in what follows, given two modules $X$ and $Y$,

$$
\tau_{(X, Y)}: X \otimes Y \cong Y \otimes X
$$

denotes the twist isomorphism. For the rest of this section, $A$ will denote an $H$ comodule algebra with structure map $\rho$.

In general, for $n \geqslant 2$, the module $A^{\otimes n}$ becomes an $H$-comodule whose structure 
map

$$
\rho^{n}: A^{\otimes n} \rightarrow H \otimes A^{\otimes n}
$$

is defined by the commutative diagram:

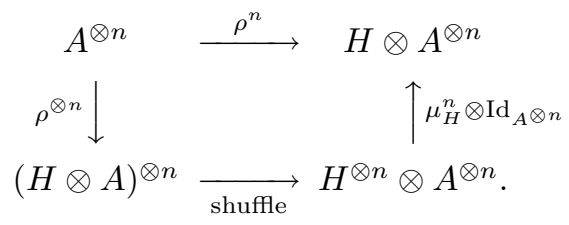

Here

$$
\mu_{H}^{n}: H^{\otimes n} \rightarrow H
$$

is the multiplication map defined by

$$
\mu_{H}^{n}\left(x_{1, n}\right)=x_{1} \cdots x_{n}
$$

for $x_{i} \in H$.

\section{2. $H^{\otimes q}$ as an $H$-bimodule}

For $q \geqslant 2$, the module $H^{\otimes q}$ is an $H$-bimodule whose left and right $H$-actions are defined by

$$
\begin{aligned}
& \mu_{l, H}^{q}\left(x, y_{1} \otimes \cdots \otimes y_{q}\right)=\sum_{(x)} x_{(1)} y_{1} \otimes \cdots \otimes x_{(q)} y_{q} \\
& \mu_{r, H}^{q}\left(y_{1} \otimes \cdots \otimes y_{q}, x\right)=\sum_{(x)} y_{1} x_{(1)} \otimes \cdots \otimes y_{q} x_{(q)}
\end{aligned}
$$

for $x, y_{1}, \ldots, y_{q} \in H$.

\section{3. $H^{\otimes q} \otimes A$ as an $A$-bimodule}

For each $q \geqslant 1$, the module $H^{\otimes q} \otimes A$ becomes an $A$-bimodule via the structure maps

$$
\begin{aligned}
& \left(\mu_{l, H}^{q} \otimes \mu_{A}\right) \circ\left(\operatorname{Id}_{H} \otimes \tau_{(A, H \otimes q)} \otimes A\right) \circ\left(\rho \otimes \operatorname{Id}_{H \otimes q \otimes A}\right): A \otimes\left(H^{\otimes q} \otimes A\right) \rightarrow H^{\otimes q} \otimes A,
\end{aligned}
$$

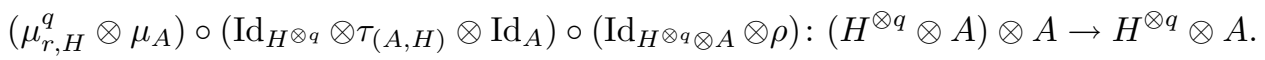

In particular, we can consider the Hochschild cochain complex $\operatorname{Hoch}^{*}\left(A, H^{\otimes q} \otimes A\right)$.

\subsection{Comodule algebra deformation bicomplex}

The deformation bicomplex of the $H$-comodule algebra $A$ is the first quadrant, cohomological bicomplex

$$
C_{\mathrm{CA}}^{* *}(A)=\left\{C_{\mathrm{CA}}^{p, q}(A), d^{p, q},(-1)^{p+1} b^{p, q}\right\}
$$

with

$$
C_{\mathrm{CA}}^{p, q}(A)= \begin{cases}0 & \text { if } p=0 \\ \operatorname{Der}(A) & \text { if }(p, q)=(1,0) \\ \operatorname{Hom}\left(A^{\otimes p}, H^{\otimes q} \otimes A\right) & \text { otherwise. }\end{cases}
$$


In the $q$ th row for $q \geqslant 0$, define the horizontal differential as the Hochschild coboundary,

$$
d^{*, q} \stackrel{\text { def }}{=} \delta_{h}^{*}: \operatorname{Hoch}^{*}\left(A, H^{\otimes q} \otimes A\right) \rightarrow \operatorname{Hoch}^{*+1}\left(A, H^{\otimes q} \otimes A\right) .
$$

For $p \geqslant 1$, the vertical differential

$$
(-1)^{p+1} b^{p, q}: \operatorname{Hom}\left(A^{\otimes p}, H^{\otimes q} \otimes A\right) \rightarrow \operatorname{Hom}\left(A^{\otimes p}, H^{\otimes(q+1)} \otimes A\right)
$$

is defined by the alternating sum

$$
b^{p, q}=\sum_{i=0}^{q+1}(-1)^{i} b^{p, q}[i],
$$

where

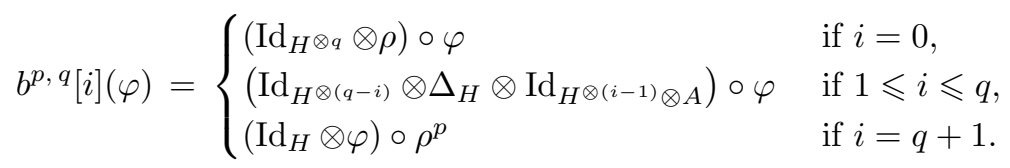

An analogue of Theorem 3.1 holds in the comodule algebra context. The total complex of the deformation bicomplex $C_{\mathrm{CA}}^{* *}(A)$ is denoted by $C_{\mathrm{CA}}^{*}(A)$ and is called the deformation complex of $A$. The $n$th cohomology module of $C_{\mathrm{CA}}^{*}(A)$ is denoted by $H_{\mathrm{CA}}^{n}(A)$.

Note that the cochain complex $\mathcal{F}_{c a}^{*}(A)$ constructed in $[\mathbf{2 0}]$ is the total complex of the sub-bicomplex of $C_{\mathrm{CA}}^{* *}(A)$ consisting of the first column $C_{\mathrm{CA}}^{1, *}(A)$ and the first row $C_{\mathrm{CA}}^{*, 1}(A)$.

\subsection{Cup product}

The module $H^{\otimes q} \otimes A$ is an associative algebra whose multiplication map is defined as

$$
\mu_{H \otimes q} \otimes A \stackrel{\text { def }}{=}\left(\mu_{H \otimes q} \otimes \mu_{A}\right) \circ\left(\operatorname{Id}_{H \otimes q} \otimes \tau_{(A, H \otimes q} \otimes \operatorname{Id}_{A}\right)
$$

where

$$
\mu_{H \otimes q}\left(x_{1} \otimes \cdots \otimes x_{q}, y_{1} \otimes \cdots \otimes y_{q}\right)=x_{1} y_{1} \otimes \cdots \otimes x_{q} y_{q}
$$

for $x_{i}, y_{j} \in H$. The three conditions in (3) hold in this case. It follows that each row

$$
C_{\mathrm{CA}}^{*, q}(A)=\operatorname{Hoch}^{*}\left(A, H^{\otimes q} \otimes A\right)
$$

admits a Hochschild $\cup$-product as in (4). Explicitly, given $\varphi \in C_{\mathrm{CA}}^{r, q}(A)$ and $\psi \in$ $C_{\mathrm{CA}}^{s, q}(A)$, their $\cup$-product is given by

$$
\varphi \cup \psi=\mu_{H \otimes q \otimes A} \circ(\varphi \otimes \psi) .
$$

\subsection{Comodule algebra deformation}

A deformation of $A$ is a power series $\Theta=\sum_{n \geqslant 0} \theta_{n} t^{n}$ with $\theta_{0}=\left(\rho, \mu_{A}\right)$ and each $\theta_{n}=\left(\rho_{n}, \pi_{n}\right) \in C_{\mathrm{CA}}^{2}(A)$, satisfying the following three conditions. Writing

$$
R=\sum_{n \geqslant 0} \rho_{n} t^{n} \quad\left(\rho_{0}=\rho\right)
$$


and

$$
\Pi=\sum_{n \geqslant 0} \pi_{n} t^{n} \quad\left(\pi_{0}=\mu_{A}\right)
$$

the three conditions are:

$$
\begin{aligned}
\left(\operatorname{Id}_{H} \otimes R\right) \circ R & =\left(\Delta_{H} \otimes \operatorname{Id}_{A}\right) \circ R, \\
R \circ \Pi & =\left(\mu_{H} \otimes \Pi\right) \circ\left(\operatorname{Id}_{H} \otimes \tau_{(A, H)} \otimes \operatorname{Id}_{A}\right) \circ R^{\otimes 2}, \\
\Pi(\Pi(a, b), c) & =\Pi(a, \Pi(b, c))
\end{aligned}
$$

for $a, b, c \in A$. Such a deformation is also denoted by $\Theta=(R, \Pi)$. The linear coefficient $\theta_{1} \in C_{\mathrm{CA}}^{2}(A)$ is called the infinitesimal. The trivial deformation is the deformation $\Theta=\theta_{0}=\left(\rho, \mu_{A}\right)$.

A formal automorphism of $A$ is a power series $\Phi=\sum_{n \geqslant 0} \phi_{n} t^{n}$ with $\phi_{0}=\operatorname{Id}_{A}$ and each $\phi_{n} \in \operatorname{End}(A)$ such that the first non-zero $\phi_{n}(n \geqslant 1)$ is a derivation on $A$.

Two deformations $\Theta=(R, \Pi)$ and $\bar{\Theta}=(\bar{R}, \bar{\Pi})$ are said to be equivalent if and only if there exists a formal automorphism $\Phi$ such that the following two conditions are satisfied:

$$
\begin{aligned}
& \bar{R}=\left(\operatorname{Id}_{H} \otimes \Phi^{-1}\right) \circ R \circ \Phi, \\
& \bar{\Pi}=\Phi^{-1} \circ \Pi \circ \Phi^{\otimes 2} .
\end{aligned}
$$

The $H$-comodule algebra $A$ is said to be rigid if every deformation of $A$ is equivalent to the trivial deformation.

The following result is the comodule algebra analogue of Theorem 5.1.

Theorem 7.1. Let $A$ be an $H$-comodule algebra. Then the following statements hold.

1. The infinitesimal $\theta_{1}$ of any deformation $\Theta$ of $A$ is a 2-cocycle in the deformation complex $C_{\mathrm{CA}}^{2}(A)$ whose cohomology class is determined by the equivalence class of $\Theta$.

2. If $H_{\mathrm{CA}}^{2}(A)=0$, then $A$ is rigid.

\section{Deformation bicomplex of comodule coalgebras}

The purposes of this section are to construct the deformation bicomplex

$$
C_{\mathrm{CC}}^{* *}(A)=\operatorname{Hom}\left(A, H^{\otimes *} \otimes A^{\otimes *}\right)
$$

of an $H$-comodule coalgebra $A$ and to list the corresponding deformation results. Deformations are taken with respect to both the $H$-comodule structure and the coalgebra structure on $A$.

\subsection{Comodule coalgebra}

For this section, let $A=\left(A, \Delta_{A}\right)$ be a coassociative coalgebra. An $H$-comodule coalgebra structure on $A$ consists of an $H$-comodule structure $\rho \in \operatorname{Hom}(A, H \otimes A)$ on $A$ such that the comultiplication map $\Delta_{A}$ is an $H$-comodule morphism, i.e.,

$$
\left(\operatorname{Id}_{H} \otimes \Delta_{A}\right) \circ \rho=\left(\mu_{H} \otimes \operatorname{Id}_{A \otimes 2}\right) \circ\left(\operatorname{Id}_{H} \otimes \tau_{(A, H)} \otimes \operatorname{Id}_{A}\right) \circ \rho^{\otimes 2} \circ \Delta_{A} .
$$

For the rest of this section, $A$ will denote an $H$-comodule coalgebra with structure map $\rho$. 


\subsection{Comodule coalgebra deformation bicomplex}

The deformation bicomplex of $A$ is the first quadrant, cohomological bicomplex

$$
C_{\mathrm{CC}}^{* *}(A)=\left\{C_{\mathrm{CC}}^{p, q}(A), d^{p, q},(-1)^{p+1} b^{p, q}\right\}
$$

with

$$
C_{\mathrm{CC}}^{p, q}(A)= \begin{cases}0 & \text { if } p=0, \\ \operatorname{Coder}(A) & \text { if }(p, q)=(1,0), \\ \operatorname{Hom}\left(A, H^{\otimes q} \otimes A^{\otimes p}\right) & \text { otherwise. }\end{cases}
$$

For $p \geqslant 1$, the vertical differential

$$
(-1)^{p+1} b^{p, q}: \operatorname{Hom}\left(A, H^{\otimes q} \otimes A^{\otimes p}\right) \rightarrow \operatorname{Hom}\left(A, H^{\otimes(q+1)} \otimes A^{\otimes p}\right)
$$

is defined by the alternating sum

$$
b^{p, q}=\sum_{i=0}^{q+1}(-1)^{i} b^{p, q}[i],
$$

where

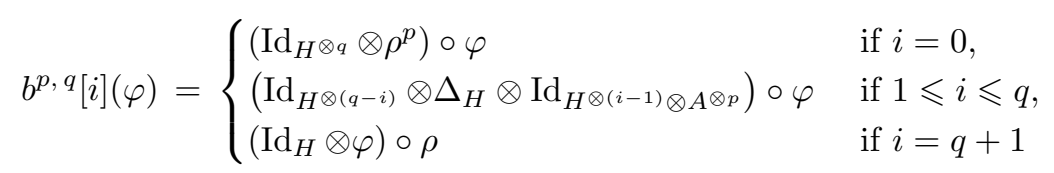

for $\varphi \in \operatorname{Hom}\left(A, H^{\otimes q} \otimes A^{\otimes p}\right)$. Here $\rho^{p}$ is the $H$-comodule structure map on $A^{\otimes p}$ defined in Section 7.1.

In the 0th row $C_{\mathrm{CC}}^{*, 0}(A)$, define the horizontal differential as the Hochschild coalgebra coboundary,

$$
d^{p, 0} \stackrel{\text { def }}{=} \delta_{c}^{p}: \operatorname{Hoch}_{c}^{p}(A, A) \rightarrow \operatorname{Hoch}_{c}^{p+1}(A, A) .
$$

In the $q$ th row $C_{\mathrm{CC}}^{*, q}(A)$ for $q \geqslant 1$, define the horizontal differential as the alternating sum

$$
d^{p, q}=\sum_{i=0}^{p+1}(-1)^{i} d^{p, q}[i]
$$

where

$$
\begin{aligned}
& d^{p, q}[i](\varphi)=
\end{aligned}
$$

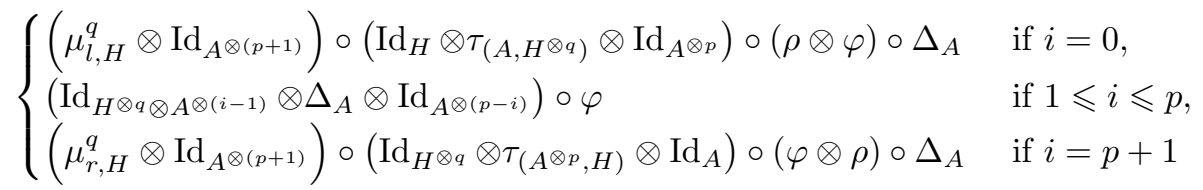

for $\varphi \in \operatorname{Hom}\left(A, H^{\otimes q} \otimes A^{\otimes p}\right)$. Here $\mu_{*, H}^{q}(*=l, r)$ are the $H$-bimodule structure maps on $H^{\otimes q}$ defined in Section 7.2.

The comodule coalgebra analogue of Theorem 3.1 holds. The total complex of the deformation bicomplex $C_{\mathrm{CC}}^{* *}(A)$ is denoted by $C_{\mathrm{CC}}^{*}(A)$ and is called the deformation complex of $A$. The $n$th cohomology module of $C_{\mathrm{CC}}^{*}(A)$ is denoted by $H_{\mathrm{CC}}^{n}(A)$. 
Note that the cochain complex $\mathcal{F}_{c c}^{*}(A)$ constructed in [20] is the total complex of the sub-bicomplex of $C_{\mathrm{CC}}^{* *}(A)$ consisting of the first column $C_{\mathrm{CC}}^{1, *}(A)$ and the first row $C_{\mathrm{CC}}^{*, 1}(A)$.

\subsection{Cup product}

For each $q \geqslant 0$, the $q$ th row $\left(C_{\mathrm{CC}}^{*, q}(A), d^{*, q}\right)$ is a DGA whose product is defined as

$$
\varphi \cup \psi \stackrel{\text { def }}{=}\left(\mu_{H \otimes q} \otimes \operatorname{Id}_{A \otimes(r+s)}\right) \circ\left(\operatorname{Id}_{H^{\otimes q}} \otimes \tau_{\left(A^{\otimes r}, H \otimes q\right.} \otimes \operatorname{Id}_{A \otimes s}\right) \circ(\varphi \otimes \psi) \circ \Delta_{A}
$$

for $\varphi \in C_{\mathrm{CC}}^{r, q}(A)$ and $\psi \in C_{\mathrm{CC}}^{s, q}(A)$.

\subsection{Comodule coalgebra deformation}

A deformation of $A$ is a power series $\Theta=\sum_{n \geqslant 0} \theta_{n} t^{n}$ with $\theta_{0}=\left(\rho, \Delta_{A}\right)$ and each $\theta_{n}=\left(\rho_{n}, \Delta_{n}\right) \in C_{\mathrm{CC}}^{2}(A)$, satisfying the following three conditions. Writing

$$
R=\sum_{n \geqslant 0} \rho_{n} t^{n} \quad\left(\rho_{0}=\rho\right)
$$

and

$$
D=\sum_{n \geqslant 0} \Delta_{n} t^{n} \quad\left(\Delta_{0}=\Delta_{A}\right),
$$

the three conditions are:

$$
\begin{aligned}
\left(\operatorname{Id}_{H} \otimes R\right) \circ R & =\left(\Delta_{H} \otimes \operatorname{Id}_{A}\right) \circ R \\
\left(\operatorname{Id}_{H} \otimes D\right) \circ R & =\left(\mu_{H} \otimes \operatorname{Id}_{A \otimes 2}\right) \circ\left(\operatorname{Id}_{H} \otimes \tau_{(A, H)} \otimes \operatorname{Id}_{A}\right) \circ R^{\otimes 2} \circ D, \\
\left(D \otimes \operatorname{Id}_{A}\right) \circ D & =\left(\operatorname{Id}_{A} \otimes D\right) \circ D .
\end{aligned}
$$

Such a deformation is also denoted by $\Theta=(R, D)$. The linear coefficient $\theta_{1} \in C_{\mathrm{CC}}^{2}(A)$ is called the infinitesimal. The trivial deformation is the deformation $\Theta=\theta_{0}=$ $\left(\rho, \Delta_{A}\right)$.

A formal automorphism of $A$ is a power series $\Phi=\sum_{n \geqslant 0} \phi_{n} t^{n}$ with $\phi_{0}=\operatorname{Id}_{A}$ and each $\phi_{n} \in \operatorname{End}(A)$ such that the first non-zero $\phi_{n}(n \geqslant 1)$ is a coderivation on $A$.

Two deformations $\Theta=(R, D)$ and $\bar{\Theta}=(\bar{R}, \bar{D})$ are said to be equivalent if and only if there exists a formal automorphism $\Phi$ such that the following two conditions are satisfied:

$$
\begin{aligned}
& \bar{R}=\left(\operatorname{Id}_{H} \otimes \Phi^{-1}\right) \circ R \circ \Phi, \\
& \bar{D}=\left(\Phi^{-1}\right)^{\otimes 2} \circ D \circ \Phi .
\end{aligned}
$$

The $H$-comodule coalgebra $A$ is said to be rigid if every deformation of $A$ is equivalent to the trivial deformation.

The following result is the comodule coalgebra analogue of Theorem 5.1.

Theorem 8.1. Let $A$ be an $H$-comodule coalgebra. Then the following statements hold.

1. The infinitesimal $\theta_{1}$ of any deformation $\Theta$ of $A$ is a 2-cocycle in the deformation complex $C_{\mathrm{CC}}^{2}(A)$ whose cohomology class is determined by the equivalence class of $\Theta$.

2. If $H_{\mathrm{CC}}^{2}(A)=0$, then $A$ is rigid. 


\section{Deformation tricomplex of module bialgebras}

In this section, we construct the deformation tricomplex

$$
C_{\mathrm{MB}}^{* * *}(A)=\operatorname{Hoch}^{*}\left(H, \operatorname{Hom}\left(A^{\otimes *}, A^{\otimes *}\right)\right)
$$

of an $H$-module bialgebra $A$ and list the corresponding deformation results. Both the $H$-module structure and the bialgebra structure on $A$ are deformed. This deformation tricomplex contains the deformation bicomplexes $C_{\mathrm{MA}}^{* *}(A)$ and $C_{\mathrm{MC}}^{* *}(A)$ for module (co)algebras and the Gerstenhaber-Schack bicomplex $C_{\mathrm{GS}}^{* *}(A)[\mathbf{7}, \mathbf{8}]$ for a bialgebra. Unlike the (co)module (co)algebra deformation bicomplexes, each cochain complex obtained from $C_{\mathrm{MB}}^{* * *}(A)$ by fixing two of the three dimensions is a DGA. In fact, each such cochain complex is either a Hochschild (coalgebra) cochain complex or is isomorphic to one. The relevant products can be identified with the Hochschild (coalgebra) $\cup$-products.

For the rest of this paper, let $A=\left(A, \mu_{A}, \Delta_{A}\right)$ be a bialgebra with associative multiplication $\mu_{A}$ and coassociative comultiplication $\Delta_{A}$.

\subsection{Biderivation}

A biderivation on $A$ is a linear self-map $\varphi \in \operatorname{End}(A)$ that is both a derivation and a coderivation on $A$. The set of biderivations on $A$ is denoted by $\operatorname{Bider}(A)$, and it is considered as a submodule of $\operatorname{End}(A)$.

\subsection{Module bialgebra}

An $H$-module bialgebra structure on $A$ is an $H$-module structure $\lambda \in$ $\operatorname{Hom}(H, \operatorname{End}(A))$ on $A$ that makes $A$ into an $H$-module algebra and an $H$-module coalgebra simultaneously. For the rest of this section, let $A$ be an $H$-module bialgebra with structure map $\lambda$.

For example, let $G_{1}$ and $G_{2}$ be two groups. Then any group homomorphism

$$
\phi: G_{1} \rightarrow \operatorname{Aut}\left(G_{2}\right)
$$

gives rise to a $K\left[G_{1}\right]$-module bialgebra structure on the group bialgebra $K\left[G_{2}\right]$ via the action

$$
\lambda(x)(y)=\phi(x)(y)
$$

for $x \in G_{1}$ and $y \in G_{2}$ [1, Example 3.6]. Similarly, suppose that $L_{1}$ and $L_{2}$ are Lie algebras and that

$$
\phi: L_{1} \rightarrow \operatorname{Der}\left(L_{2}\right)
$$

is a Lie algebra morphism. Then the same formula $(22)$ gives rise to a $U\left(L_{1}\right)$-module bialgebra structure on the enveloping bialgebra $U\left(L_{2}\right)$ [1, Example 3.7].

\section{3. $H$-bimodule structure on $\operatorname{Hom}\left(\boldsymbol{A}^{\otimes p}, \boldsymbol{A}^{\otimes q}\right)$}

When $A$ is an $H$-module bialgebra, there is an $H$-bimodule structure on the module $\operatorname{Hom}\left(A^{\otimes p}, A^{\otimes q}\right)$ for $p, q \geqslant 1$. The left and right $H$-actions are given as follows:

$$
\begin{aligned}
& (x \varphi)\left(a_{1, p}\right)=\sum \lambda\left(x_{(1)}\right)\left(\varphi\left(a_{1, p}\right)^{1}\right) \otimes \cdots \otimes \lambda\left(x_{(q)}\right)\left(\varphi\left(a_{1, p}\right)^{q}\right), \\
& (\varphi x)\left(a_{1, p}\right)=\sum \varphi\left(\lambda\left(x_{(1)}\right)\left(a_{1}\right) \otimes \cdots \otimes \lambda\left(x_{(p)}\right)\left(a_{p}\right)\right) .
\end{aligned}
$$


Here $x \in H, \varphi \in \operatorname{Hom}\left(A^{\otimes p}, A^{\otimes q}\right), a_{i} \in A$, and

$$
\varphi\left(a_{1, p}\right)=\sum \varphi\left(a_{1, p}\right)^{1} \otimes \cdots \otimes \varphi\left(a_{1, p}\right)^{q} .
$$

This generalizes the constructions in Sections 3.1 and 6.3. In particular, we can consider the Hochschild cochain complex $\operatorname{Hoch}^{*}\left(H, \operatorname{Hom}\left(A^{\otimes p}, A^{\otimes q}\right)\right)$.

\subsection{Module bialgebra deformation tricomplex}

The deformation tricomplex of the $H$-module bialgebra $A$ is the first octant, cohomological tricomplex

$$
C_{\mathrm{MB}}^{* * *}(A)=\left\{C_{\mathrm{MB}}^{p, q, r}(A),(-1)^{q+1} d_{I}^{p, q, r},(-1)^{r+1} d_{I I}^{p, q, r},(-1)^{p+1} d_{I I I}^{p, q, r}\right\}
$$

with

$$
C_{\mathrm{MB}}^{p, q, r}(A)= \begin{cases}0 & \text { if } p=0 \text { or } q=0, \\ \operatorname{Bider}(A) & \text { if }(p, q, r)=(1,1,0), \\ \operatorname{Hom}\left(H^{\otimes r}, \operatorname{Hom}\left(A^{\otimes p}, A^{\otimes q}\right)\right) & \text { otherwise. }\end{cases}
$$

The differential

$$
(-1)^{q+1} d_{I}^{p, q, r}: C_{\mathrm{MB}}^{p, q, r}(A) \rightarrow C_{\mathrm{MB}}^{p+1, q, r}(A)
$$

is defined using the alternating sum

$$
d_{I}^{p, q, r}=\sum_{i=0}^{p+1}(-1)^{i} d_{I}^{p, q, r}[i]
$$

where

$$
\begin{aligned}
& \left(d_{I}^{p, q, r}[i] \varphi\right)\left(x_{1, r}\right)\left(a_{1, p+1}\right)= \\
& \begin{cases}\sum \mu_{l, A}^{q}\left(\lambda\left(x_{1(1)} \cdots x_{r(1)}\right)\left(a_{1}\right), \varphi\left(x_{1(2)} \otimes \cdots \otimes x_{r(2)}\right)\left(a_{2, p+1}\right)\right) & \text { if } i=0, \\
\varphi\left(x_{1, r}\right)\left(a_{1, i-1} \otimes\left(a_{i} a_{i+1}\right) \otimes a_{i+2, p+1}\right) & \text { if } 1 \leqslant i \leqslant p, \\
\sum \mu_{r, A}^{q}\left(\varphi\left(x_{1(1)} \otimes \cdots \otimes x_{r(1)}\right)\left(a_{1, p}\right), \lambda\left(x_{1(2)} \cdots x_{r(2)}\right)\left(a_{p+1}\right)\right) & \text { if } i=p+1\end{cases}
\end{aligned}
$$

for $\varphi \in C_{\mathrm{MB}}^{p, q, r}(A), x_{k} \in H$, and $a_{l} \in A$. Here $\mu_{l, A}^{q}$ and $\mu_{r, A}^{q}$ are the left and right actions of $A$ on $A^{\otimes q}$, defined as in Section 7.2.

The differential

$$
(-1)^{r+1} d_{I I}^{p, q, r}: C_{\mathrm{MB}}^{p, q, r}(A) \rightarrow C_{\mathrm{MB}}^{p, q+1, r}(A)
$$

is defined using the alternating sum

$$
d_{I I}^{p, q, r}=\sum_{i=0}^{q+1}(-1)^{i} d_{I I}^{p, q, r}[i]
$$


where

$$
\begin{aligned}
& \left(d_{I I}^{p, q, r}[i] \varphi\right)\left(x_{1, r}\right)\left(a_{1, p}\right)= \\
& \qquad \begin{array}{cl}
\sum \lambda\left(x_{1(1)} \cdots x_{r(1)}\right)\left(a_{1(1)} \cdots a_{p(1)}\right) & \\
\otimes \varphi\left(x_{1(2)} \otimes \cdots \otimes x_{r(2)}\right)\left(a_{1(2)} \otimes \cdots \otimes a_{p(2)}\right) & \text { if } i=0, \\
\left(\operatorname{Id}_{A \otimes(i-1)} \otimes \Delta_{A} \otimes \operatorname{Id}_{A((-i)}\right)\left(\varphi\left(x_{1, r}\right)\left(a_{1, p}\right)\right) & \text { if } 1 \leqslant i \leqslant q, \\
\sum \varphi\left(x_{1(1)} \otimes \cdots \otimes x_{r(1)}\right)\left(a_{1(1)} \otimes \cdots \otimes a_{p(1)}\right) & \\
\otimes \lambda\left(x_{1(2)} \cdots x_{r(2)}\right)\left(a_{1(2)} \cdots a_{p(2)}\right) & \text { if } i=q+1 .
\end{array}
\end{aligned}
$$

Finally, the differential

$$
(-1)^{p+1} d_{I I I}^{p, q, r}: C_{\mathrm{MB}}^{p, q, r}(A) \rightarrow C_{\mathrm{MB}}^{p, q, r+1}(A)
$$

is defined using the Hochschild coboundary

$$
d_{I I I}^{p, q, r} \stackrel{\text { def }}{=} \delta_{h}^{r}: \operatorname{Hoch}^{r}\left(H, \operatorname{Hom}\left(A^{\otimes p}, A^{\otimes q}\right)\right) \rightarrow \operatorname{Hoch}^{r+1}\left(H, \operatorname{Hom}\left(A^{\otimes p}, A^{\otimes q}\right)\right) .
$$

In particular, $d_{I I I}^{p, q, r}$ is the alternating sum

$$
d_{I I I}^{p, q, r}=\sum_{i=0}^{r+1}(-1)^{i} d_{I I I}^{p, q, r}[i]
$$

where

$$
d_{I I I}^{p, q, r}[i]=\delta_{h}^{r}[i]
$$

as in (2).

The following observations, which are the module bialgebra analogue of Theorem 3.1, ensure that $C_{\mathrm{MB}}^{* * *}(A)$ is indeed a tricomplex.

Theorem 9.1. Let $A$ be an $H$-module bialgebra. Then:

1. The following statements hold for all possible values of $p, q, r$, and $k<l$ :

$$
\begin{aligned}
& d_{I}^{p+1, q, r}[l] \circ d_{I}^{p, q, r}[k]=d_{I}^{p+1, q, r}[k] \circ d_{I}^{p, q, r}[l-1], \\
& d_{I I}^{p, q+1, r}[l] \circ d_{I I}^{p, q, r}[k]=d_{I I}^{p, q+1, r}[k] \circ d_{I I}^{p, q, r}[l-1], \\
& d_{I I I}^{p, q, r+1}[l] \circ d_{I I I}^{p, q, r}[k]=d_{I I I}^{p, q, r+1}[k] \circ d_{I I I}^{p, q, r}[l-1] .
\end{aligned}
$$

2. The following statements hold for all possible values of $p, q, r, i$, and $j$ :

$$
\begin{aligned}
& d_{I I}^{p+1, q, r}[j] \circ d_{I}^{p, q, r}[i]=d_{I}^{p, q+1, r}[i] \circ d_{I I}^{p, q, r}[j], \\
& d_{I I}^{p, q, r+1}[j] \circ d_{I I I}^{p, q, r}[i]=d_{I I I}^{p, q+1, r}[i] \circ d_{I I}^{p, q, r}[j], \\
& d_{I I I}^{p+1, q, r}[j] \circ d_{I}^{p, q, r}[i]=d_{I}^{p, q, r+1}[i] \circ d_{I I I}^{p, q, r}[j] .
\end{aligned}
$$

In particular, every two-dimensional plane in $C_{\mathrm{MB}}^{* * *}(A)$ is a bicomplex.

\subsection{Boundary planes}

Observe that the boundary planes of the deformation tricomplex $C_{\mathrm{MB}}^{* * *}(A)$ are either known or have been discussed in previous sections. In fact:

1. The $p=1$ plane $C_{\mathrm{MB}}^{1, *, *}(A)$ coincides with the deformation bicomplex $C_{\mathrm{MC}}^{* *}(A)$ in which $A$ is regarded as an $H$-module coalgebra (see Section 6). 
2. The $q=1$ plane $C_{\mathrm{MB}}^{*, 1, *}(A)$ coincides with the deformation bicomplex $C_{\mathrm{MA}}^{* *}(A)$ in which $A$ is regarded as an $H$-module algebra (see Section 3 ).

3. The $r=0$ plane $C_{\mathrm{MB}}^{*, *, 0}(A)$ coincides with the Gerstenhaber-Schack deformation bicomplex $C_{\mathrm{GS}}^{* *}(A)$ (denoted by $\widehat{C}_{b}^{\bullet}, \bullet(A, A)$ in $[\mathbf{7}, \mathbf{8}]$ ) in which $A$ is regarded as only a bialgebra.

As in the (co)module (co)algebra cases, the only exception to the above remarks is the entry $C_{\mathrm{MB}}^{1,1,0}(A)=\operatorname{Bider}(A)$.

\subsection{Cup products}

Each cochain complex in the deformation tricomplex $C_{\mathrm{MB}}^{* * *}(A)$ has an associative $\cup$-product that makes it into a DGA.

In the direction of $d_{I}$, fix $q \geqslant 1$ and $r \geqslant 0$. Then the cochain complex $C_{\mathrm{MB}}^{*, q, r}(A)$ is a DGA whose product is defined as

$$
\begin{aligned}
& (\varphi \cup \psi)\left(x_{1, r}\right)\left(a_{1, p_{1}+p_{2}}\right) \stackrel{\text { def }}{=} \\
& \quad \mu_{A \otimes q}\left(\varphi\left(x_{1(1)} \otimes \cdots \otimes x_{r(1)}\right)\left(a_{1, p_{1}}\right), \psi\left(x_{1(2)} \otimes \cdots \otimes x_{r(2)}\right)\left(a_{p_{1}+1, p_{1}+p_{2}}\right)\right)
\end{aligned}
$$

for $\varphi \in C_{\mathrm{MB}}^{p_{1}, q, r}(A)$ and $\psi \in C_{\mathrm{MB}}^{p_{2}, q, r}(A)$. This generalizes the $\cup$-product in $C_{\mathrm{MA}}^{*, r}(A)$ discussed in Section 4 .

In fact, it can be identified with a Hochschild $\cup$-product. There is a canonical isomorphism of cochain complexes,

$$
\left(C_{\mathrm{MB}}^{*, q, r}(A),(-1)^{q+1} d_{I}^{*, q, r}\right) \cong(-1)^{q+1}\left(\operatorname{Hoch}^{*}\left(A, \operatorname{Hom}\left(H^{\otimes r}, A^{\otimes q}\right)\right), \delta_{h}\right)
$$

given by the Hom $-\otimes$ adjunction (twice). The left and right actions of $A$ on $\operatorname{Hom}\left(H^{\otimes r}, A^{\otimes q}\right)$ are given by

$$
\begin{aligned}
& (a f)\left(x_{1, r}\right)=\sum \mu_{l, A}^{q}\left(\lambda\left(x_{1(1)} \cdots x_{r(1)}\right)(a), f\left(x_{1(2)} \otimes \cdots \otimes x_{r(2)}\right)\right), \\
& (f a)\left(x_{1, r}\right)=\sum \mu_{r, A}^{q}\left(f\left(x_{1(1)} \otimes \cdots \otimes x_{r(1)}\right), \lambda\left(x_{1(2)} \cdots x_{r(2)}\right)(a)\right)
\end{aligned}
$$

for $a \in A, f \in \operatorname{Hom}\left(H^{\otimes r}, A^{\otimes q}\right)$, and $x_{i} \in H$. The module $\operatorname{Hom}\left(H^{\otimes r}, A^{\otimes q}\right)$ is an associative algebra via the product

$$
(f \cdot g)\left(x_{1, r}\right)=\mu_{A \otimes q}\left(f\left(x_{1(1)} \otimes \cdots \otimes x_{r(1)}\right), g\left(x_{1(2)} \otimes \cdots \otimes x_{r(2)}\right)\right)
$$

such that the conditions (3) are satisfied. The resulting Hochschild $\cup$-product corresponds, via the isomorphism (24), to the $\cup$-product defined in (23).

In the direction of $d_{I I}$, fix $p \geqslant 1$ and $r \geqslant 0$. Then the cochain complex $C_{\mathrm{MB}}^{p, *, r}(A)$ is a DGA whose product is defined as

$$
\begin{aligned}
(\varphi \cup \psi)\left(x_{1, r}\right)\left(a_{1, p}\right) \stackrel{\text { def }}{=} \sum \varphi\left(x_{1(1)} \otimes \cdots \otimes x_{r(1)}\right)\left(a_{1(1)} \otimes \cdots \otimes a_{p(1)}\right) \otimes \\
\psi\left(x_{1(2)} \otimes \cdots \otimes x_{r(2)}\right)\left(a_{1(2)} \otimes \cdots \otimes a_{p(2)}\right)
\end{aligned}
$$

for $\varphi \in C_{\mathrm{MB}}^{p, q_{1}, r}(A)$ and $\psi \in C_{\mathrm{MB}}^{p, q_{2}, r}(A)$. This generalizes the $\cup$-product in $C_{\mathrm{MC}}^{*, r}(A)$ discussed in Section 6.5.

Moreover, there is a canonical isomorphism,

$$
\left(C_{\mathrm{MB}}^{p, *, r}(A),(-1)^{r+1} d_{I I}^{p, *, r}\right) \cong(-1)^{r+1}\left(\operatorname{Hoch}_{c}^{*}\left(H^{\otimes r} \otimes A^{\otimes p}, A\right), \delta_{c}\right)
$$

of cochain complexes given by the Hom $-\otimes$ adjunction. The left and right $A$-coactions 
on $H^{\otimes r} \otimes A^{\otimes p}$ are given by

$$
\begin{aligned}
& x_{1, r} \otimes a_{1, p} \mapsto \lambda\left(x_{1(1)} \cdots x_{r(1)}\right)\left(a_{1(1)} \cdots a_{p(1)}\right) \\
& \otimes\left(x_{1(2)} \otimes \cdots \otimes x_{r(2)} \otimes a_{1(2)} \otimes \cdots \otimes a_{p(2)}\right), \\
& x_{1, r} \otimes a_{1, p} \mapsto\left(x_{1(1)} \otimes \cdots \otimes x_{r(1)} \otimes a_{1(1)} \otimes \cdots \otimes a_{p(1)}\right) \\
& \otimes \lambda\left(x_{1(2)} \cdots x_{r(2)}\right)\left(a_{1(2)} \cdots a_{p(2)}\right) .
\end{aligned}
$$

The module $H^{\otimes r} \otimes A^{\otimes p}$ is a coassociative coalgebra via the coproduct

$$
\begin{aligned}
& \Delta\left(x_{1, r} \otimes a_{1, p}\right)=\sum\left(x_{1(1)} \otimes \cdots \otimes x_{r(1)} \otimes a_{1(1)} \otimes \cdots \otimes a_{p(1)}\right) \\
& \otimes\left(x_{1(2)} \otimes \cdots \otimes x_{r(2)} \otimes a_{1(2)} \otimes \cdots \otimes a_{p(2)}\right)
\end{aligned}
$$

such that the three conditions in (13) are satisfied. The resulting Hochschild coalgebra $\cup$-product corresponds, via the isomorphism (26), to the $\cup$-product defined in (25).

In the direction of $d_{I I I}$, fix $p \geqslant 1$ and $q \geqslant 1$. Note that the module $\operatorname{Hom}\left(A^{\otimes p}, A^{\otimes q}\right)$ is an associative algebra via the product

$$
f \cdot g \stackrel{\text { def }}{=} f \circ \Delta_{A}^{p-1} \circ \mu_{A}^{q} \circ g
$$

where

$$
\Delta_{A}^{0}=\operatorname{Id}_{A}=\mu_{A}^{1} .
$$

In the particular case $p=q=1$, this product is simply the composition product in $\operatorname{Hom}(A, A)$. Regarding $\operatorname{Hom}\left(A^{\otimes p}, A^{\otimes q}\right)$ as an $H$-bimodule, the three conditions in (3) hold. It follows that the Hochschild cochain complex

$$
C_{\mathrm{MB}}^{p, q * *}(A)=(-1)^{p+1} \operatorname{Hoch}^{*}\left(H, \operatorname{Hom}\left(A^{\otimes p}, A^{\otimes q}\right)\right)
$$

admits a Hochschild $\cup$-product (4) that makes it into a DGA.

\subsection{Total complex}

The total complex $C_{\mathrm{MB}}^{*}(A)$, called the deformation complex of $A$, of the deformation tricomplex $C_{\mathrm{MB}}^{* * *}(A)$ is defined as usual but with a shift of degree:

$$
C_{\mathrm{MB}}^{n}(A) \stackrel{\text { def }}{=} \bigoplus_{p+q+r=n+1} C_{\mathrm{MB}}^{p, q, r}(A) .
$$

In particular, we have that

$$
\begin{aligned}
& C_{\mathrm{MB}}^{1}(A)=\operatorname{Bider}(A), \\
& C_{\mathrm{MB}}^{2}(A)=\operatorname{Hom}(H, \operatorname{Hom}(A, A)) \oplus \operatorname{Hom}\left(A^{\otimes 2}, A\right) \oplus \operatorname{Hom}\left(A, A^{\otimes 2}\right) .
\end{aligned}
$$

The degree shift is introduced to ensure that the deformation results below have the same degree conventions as in the previous sections and as in $[\mathbf{7}, \mathbf{8}]$.

The $n$th cohomology module of the deformation complex $C_{\mathrm{MB}}^{*}(A)$ is denoted by $H_{\mathrm{MB}}^{n}(A)$.

\subsection{Module bialgebra deformation}

A deformation of $A$ as an $H$-module bialgebra is a power series $\Theta=\sum_{n \geqslant 0} \theta_{n} t^{n}$ with $\theta_{0}=\left(\lambda, \mu_{A}, \Delta_{A}\right) \in C_{\mathrm{MB}}^{2}(A)$ and each $\theta_{n}=\left(\lambda_{n}, \pi_{n}, \Delta_{n}\right) \in C_{\mathrm{MB}}^{2}(A)$, satisfying 
the five conditions for module algebra deformations (10) and module coalgebra deformations (15). Using the notations from earlier sections, such a deformation is also denoted by

$$
\Theta=(\Lambda, \Pi, D)
$$

The infinitesimal of a deformation $\Theta$ is the linear coefficient $\theta_{1}$.

A formal automorphism of $A$ is a power series $\sum_{n \geqslant 0} \phi_{n} t^{n}$ with $\phi_{0}=\operatorname{Id}_{A}$ and each $\phi_{n} \in \operatorname{End}(A)$ such that the first non-zero $\phi_{n}(n \geqslant 1)$ is a biderivation on $A$.

Two deformations $\Theta=(\Lambda, \Pi, D)$ and $\bar{\Theta}=(\bar{\Lambda}, \bar{\Pi}, \bar{D})$ are said to be equivalent if and only if there exists a formal automorphism $\Phi$ such that the three conditions in (11) and (16) are satisfied.

The trivial deformation is the deformation $\Theta=\theta_{0}=\left(\lambda, \mu_{A}, \Delta_{A}\right)$. The $H$-module bialgebra $A$ is said to be rigid if every deformation of $A$ is equivalent to the trivial deformation.

The following result is the module bialgebra analogue of Theorem 5.1.

Theorem 9.2. Let $A$ be an $H$-module bialgebra. Then the following statements hold.

1. The infinitesimal $\theta_{1}$ of any deformation $\Theta$ of $A$ is a 2-cocycle in the deformation complex $C_{\mathrm{MB}}^{2}(A)$ whose cohomology class is determined by the equivalence class of $\Theta$.

2. If $H_{\mathrm{MB}}^{2}(A)=0$, then $A$ is rigid.

\section{Deformation tricomplex of comodule bialgebras}

The purposes of this section are to construct the deformation tricomplex

$$
C_{\mathrm{CB}}^{* * *}(A)=\operatorname{Hom}\left(A^{\otimes *}, H^{\otimes *} \otimes A^{\otimes *}\right)
$$

of an $H$-comodule bialgebra $A$ and to list the corresponding deformation results. Both the $H$-comodule structure and the bialgebra structure on $A$ are deformed. This deformation tricomplex contains the deformation bicomplexes $C_{\mathrm{CA}}^{* *}(A)$ and $C_{\mathrm{CC}}^{* *}(A)$ for comodule (co)algebras and the Gerstenhaber-Schack bicomplex $C_{\mathrm{GS}}^{* *}(A)[\mathbf{7}, \mathbf{8}]$ for a bialgebra.

\subsection{Comodule bialgebra}

An $H$-comodule bialgebra structure on a bialgebra $A$ is an $H$-comodule structure $\rho \in \operatorname{Hom}(A, H \otimes A)$ that makes $A$ into an $H$-comodule algebra and an $H$-comodule coalgebra simultaneously.

For example, let $H$ be a commutative Hopf algebra with antipode $S$. Then the map $\rho \in \operatorname{Hom}(H, H \otimes H)$ defined by

$$
\rho(x)=\sum\left(x_{(1)} S x_{(3)}\right) \otimes x_{(2)}
$$

for $x \in H$ gives $H$ an $H$-comodule bialgebra structure [1, Example 3.8]. 
10.2. $\quad A$-bimodule structure on $H^{\otimes r} \otimes A^{\otimes q}$

For $r \geqslant 0$ and $q \geqslant 1$, there is an $A$-bimodule structure on the module $H^{\otimes r} \otimes A^{\otimes q}$ whose left and right $A$-action maps are defined as

$$
\begin{aligned}
\left(\mu_{l, H}^{r} \otimes \mu_{l, A}^{q}\right) \circ\left(\operatorname{Id}_{H} \otimes \tau_{\left(A, H^{\otimes r}\right.} \otimes \operatorname{Id}_{A^{\otimes q}}\right) \circ\left(\rho \otimes \operatorname{Id}_{H^{\otimes r} \otimes A}^{\otimes q}\right) & : A \otimes\left(H^{\otimes r} \otimes A^{\otimes q}\right) \\
& \rightarrow H^{\otimes r} \otimes A^{\otimes q} \\
\left(\mu_{r, H}^{r} \otimes \mu_{r, A}^{q}\right) \circ\left(\operatorname{Id}_{H^{\otimes r}} \otimes \tau_{\left(A^{\otimes q}, H\right)} \otimes \operatorname{Id}_{A}\right) \circ\left(\operatorname{Id}_{H^{\otimes r} \otimes A^{\otimes q}} \otimes \rho\right) & :\left(H^{\otimes r} \otimes A^{\otimes q}\right) \otimes A \\
& \rightarrow H^{\otimes r} \otimes A^{\otimes q}
\end{aligned}
$$

when $r \geqslant 1$. When $r=0$, the left and right $A$-action maps on $A^{\otimes q}$ are simply $\mu_{l, A}^{q}$ and $\mu_{r, A}^{q}$. In particular, we can consider the Hochschild cochain complex

$$
\operatorname{Hoch}^{*}\left(A, H^{\otimes r} \otimes A^{\otimes q}\right) .
$$

\subsection{Comodule bialgebra deformation tricomplex}

The deformation tricomplex of the $H$-comodule bialgebra $A$ is the first octant, cohomological tricomplex

$$
C_{\mathrm{CB}}^{* * *}(A)=\left\{C_{\mathrm{CB}}^{p, q, r}(A),(-1)^{q+1} d_{I}^{p, q, r},(-1)^{r+1} d_{I I}^{p, q, r},(-1)^{p+1} d_{I I I}^{p, q, r}\right\}
$$

with

$$
C_{\mathrm{CB}}^{p, q, r}(A)= \begin{cases}0 & \text { if } p=0 \text { or } q=0, \\ \operatorname{Bider}(A) & \text { if }(p, q, r)=(1,1,0), \\ \operatorname{Hom}\left(A^{\otimes p}, H^{\otimes r} \otimes A^{\otimes q}\right) & \text { otherwise. }\end{cases}
$$

The differential

$$
(-1)^{q+1} d_{I}^{p, q, r}: C_{\mathrm{CB}}^{p, q, r}(A) \rightarrow C_{\mathrm{CB}}^{p+1, q, r}(A)
$$

is defined using the Hochschild coboundary

$$
d_{I}^{p, q, r} \stackrel{\text { def }}{=} \delta_{h}^{p}: \operatorname{Hoch}^{p}\left(A, H^{\otimes r} \otimes A^{\otimes q}\right) \rightarrow \operatorname{Hoch}^{p+1}\left(A, H^{\otimes r} \otimes A^{\otimes q}\right) .
$$

The differential

$$
(-1)^{r+1} d_{I I}^{p, q, r}: C_{\mathrm{CB}}^{p, q, r}(A) \rightarrow C_{\mathrm{CB}}^{p, q+1, r}(A)
$$

is defined using the alternating sum

$$
d_{I I}^{p, q, r}=\sum_{i=0}^{q+1}(-1)^{i} d_{I I}^{p, q, r}[i]
$$

where

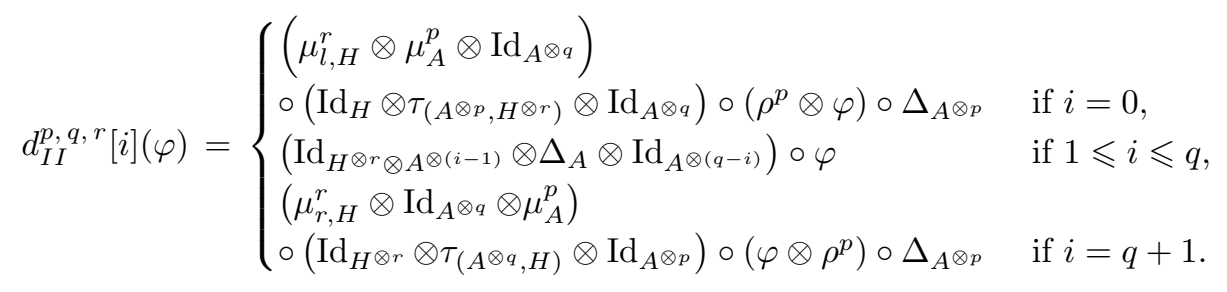


Here

$$
\rho^{p}: A^{\otimes p} \rightarrow H \otimes A^{\otimes p}
$$

and

$$
\mu_{A}^{p}: A^{\otimes p} \rightarrow A
$$

are as defined in Section 7.1 and (17), respectively. The map

$$
\Delta_{A \otimes p}: A^{\otimes p} \rightarrow A^{\otimes p} \otimes A^{\otimes p}
$$

is defined as

$$
\Delta_{A \otimes p}\left(a_{1, p}\right)=\sum\left(a_{1(1)} \otimes \cdots \otimes a_{p(1)}\right) \otimes\left(a_{1(2)} \otimes \cdots \otimes a_{p(2)}\right)
$$

for $a_{1}, \ldots, a_{p} \in A$.

Finally, the differential

$$
(-1)^{p+1} d_{I I I}^{p, q, r}: C_{\mathrm{CB}}^{p, q, r}(A) \rightarrow C_{\mathrm{CB}}^{p, q, r+1}(A)
$$

is defined using the alternating sum

$$
d_{I I I}^{p, q, r}=\sum_{i=0}^{r+1}(-1)^{i} d_{I I I}^{p, q, r}[i]
$$

where

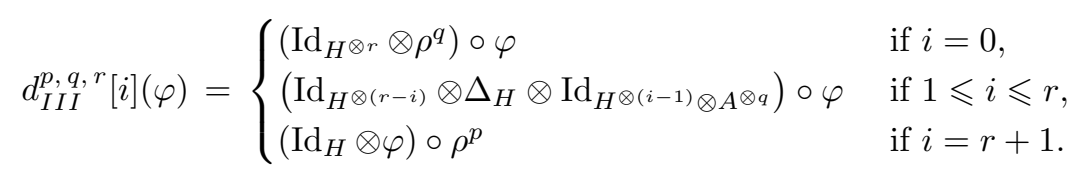

The six statements in Theorem 9.1 still hold in the comodule bialgebra context, so $C_{\mathrm{CB}}^{* * *}(A)$ is indeed a tricomplex.

\subsection{Boundary planes}

As in the module bialgebra case, the boundary planes of the deformation tricomplex $C_{\mathrm{CB}}^{* * *}(A)$ are either known or have been discussed in previous sections. In fact:

1. The $p=1$ plane $C_{\mathrm{CB}}^{1, * * *}(A)$ coincides with the deformation bicomplex $C_{\mathrm{CC}}^{* *}(A)$ in which $A$ is regarded as an $H$-comodule coalgebra (see Section 8).

2. The $q=1$ plane $C_{\mathrm{CB}}^{*, 1, *}(A)$ coincides with the deformation bicomplex $C_{\mathrm{CA}}^{* *}(A)$ in which $A$ is regarded as an $H$-comodule algebra (see Section 7).

3. The $r=0$ plane $C_{\mathrm{CB}}^{*, * 0}(A)$ coincides with the Gerstenhaber-Schack deformation bicomplex $C_{\mathrm{GS}}^{* *}(A)$ (denoted by $\widehat{C}_{b}^{\bullet}, \bullet(A, A)$ in $[\mathbf{7}, \mathbf{8}]$ ) in which $A$ is regarded as only a bialgebra.

Again, the only exception to the above remarks is the entry $C_{\mathrm{CB}}^{1,1,0}(A)=\operatorname{Bider}(A)$.

\subsection{Cup products}

Each cochain complex in the deformation tricomplex $C_{\mathrm{CB}}^{* * *}(A)$ is a DGA. 
In the direction of $d_{I}$, fix $q \geqslant 1$ and $r \geqslant 0$. Note that the module $H^{\otimes r} \otimes A^{\otimes q}$ is an associative algebra whose multiplication map is

$$
\mu_{H \otimes r \otimes A \otimes q}=\left(\mu_{H^{\otimes r}} \otimes \mu_{A \otimes q}\right) \circ\left(\operatorname{Id}_{H^{\otimes r}} \otimes \tau_{\left(A^{\otimes q}, H^{\otimes r}\right)} \otimes \operatorname{Id}_{A^{\otimes q}}\right) .
$$

Regarding $H^{\otimes r} \otimes A^{\otimes q}$ as an $A$-bimodule, the three conditions in (3) hold. It follows that

$$
C_{\mathrm{CB}}^{*, q, r}(A)=(-1)^{q+1} \operatorname{Hoch}^{*}\left(A, H^{\otimes r} \otimes A^{\otimes q}\right)
$$

admits a Hochschild $\cup$-product that makes it into a DGA. This generalizes the $\cup$ product in $C_{\mathrm{CA}}^{*, r}(A)$ discussed in Section 7.5.

In the direction of $d_{I I}$, fix $p \geqslant 1$ and $r \geqslant 0$. Then the cochain complex $C_{\mathrm{CB}}^{p, *, r}(A)$ is a DGA whose product is defined as

$$
\varphi \cup \psi \stackrel{\text { def }}{=}\left(\mu_{H \otimes r} \otimes \operatorname{Id}_{A^{\otimes\left(q_{1}+q_{2}\right)}}\right) \circ\left(\operatorname{Id}_{H^{\otimes r}} \otimes \tau_{\left(A^{\otimes q_{1}}, H^{\otimes r}\right)} \otimes \operatorname{Id}_{A^{\otimes q_{2}}}\right) \circ(\varphi \otimes \psi) \circ \Delta_{A^{\otimes p}}
$$

for $\varphi \in C_{\mathrm{CB}}^{p, q_{1}, r}(A)$ and $\psi \in C_{\mathrm{CB}}^{p, q_{2}, r}(A)$. This generalizes the $\cup$-product in $C_{\mathrm{CC}}^{*, r}(A)$ discussed in Section 8.3.

In the direction of $d_{I I I}$, fix $p \geqslant 1$ and $q \geqslant 1$. Then the cochain complex $C_{\mathrm{CB}}^{p, q, *}(A)$ is a DGA whose product is defined as

$$
\varphi \cup \psi \stackrel{\text { def }}{=}\left(\operatorname{Id}_{H^{\otimes\left(r_{1}+r_{2}\right)}} \otimes \mu_{A \otimes q}\right) \circ\left(\operatorname{Id}_{H^{\otimes r_{1}}} \otimes \tau_{\left(A^{\otimes q}, H^{\otimes r_{2}}\right)} \otimes \operatorname{Id}_{A \otimes q}\right) \circ(\varphi \otimes \psi) \circ \Delta_{A \otimes p}
$$

for $\varphi \in C_{\mathrm{CB}}^{p, q, r_{1}}(A)$ and $\psi \in C_{\mathrm{CB}}^{p, q, r_{2}}(A)$.

\subsection{Total complex}

The total complex $C_{\mathrm{CB}}^{*}(A)$, called the deformation complex of $A$, of the deformation tricomplex $C_{\mathrm{CB}}^{* * *}(A)$ is defined as usual but with a shift of degree:

$$
C_{\mathrm{CB}}^{n}(A) \stackrel{\text { def }}{=} \bigoplus_{p+q+r=n+1} C_{\mathrm{CB}}^{p, q, r}(A) .
$$

In particular, we have that

$$
\begin{aligned}
& C_{\mathrm{CB}}^{1}(A)=\operatorname{Bider}(A), \\
& C_{\mathrm{CB}}^{2}(A)=\operatorname{Hom}(A, H \otimes A) \oplus \operatorname{Hom}\left(A^{\otimes 2}, A\right) \oplus \operatorname{Hom}\left(A, A^{\otimes 2}\right) .
\end{aligned}
$$

The $n$th cohomology module of the deformation complex $C_{\mathrm{CB}}^{*}(A)$ is denoted by $H_{\mathrm{CB}}^{n}(A)$.

\subsection{Comodule bialgebra deformation}

A deformation of $A$ as an $H$-comodule bialgebra is a power series $\Theta=\sum_{n \geqslant 0} \theta_{n} t^{n}$ with $\theta_{0}=\left(\rho, \mu_{A}, \Delta_{A}\right) \in C_{\mathrm{CB}}^{2}(A)$ and each $\theta_{n}=\left(\rho_{n}, \pi_{n}, \Delta_{n}\right) \in C_{\mathrm{CB}}^{2}(A)$, satisfying the five conditions for comodule algebra deformation (18) and comodule coalgebra deformation (20). The infinitesimal of $\Theta$ is the linear coefficient $\theta_{1}$.

The notions of a formal automorphism of $A$ and equivalence of deformations are defined exactly as in the module bialgebra case, except that, in defining the latter, the conditions (19) and (21) are used.

The trivial deformation is the deformation $\Theta=\theta_{0}=\left(\rho, \mu_{A}, \Delta_{A}\right)$. The $H$-comodule bialgebra $A$ is said to be rigid if every deformation of $A$ is equivalent to the trivial deformation. 
The following result is the comodule bialgebra analogue of Theorem 5.1.

Theorem 10.1. Let $A$ be an $H$-comodule bialgebra. Then the following statements hold.

1. The infinitesimal $\theta_{1}$ of any deformation $\Theta$ of $A$ is a 2-cocycle in the deformation complex $C_{\mathrm{CB}}^{2}(A)$ whose cohomology class is determined by the equivalence class of $\Theta$.

2. If $H_{\mathrm{CB}}^{2}(A)=0$, then $A$ is rigid.

\section{Acknowledgements}

The author thanks the referee for his/her suggestions.

\section{References}

[1] E. Abe, Hopf algebras, Cambridge Tracts in Mathematics 74, Cambridge Univ. Press, Cambridge, 1977.

[2] J.M. Boardman, Stable operations in generalized cohomology, Handbook of algebraic topology, 585-686, North-Holland, 1995.

[3] S. Dăsălescu, C. Năstăsescu and Ş. Raianu, Hopf algebra: An introduction, Monographs and Textbooks in Pure and Applied Math. 235, Marcel Dekker Inc., New York, 2001.

[4] D.B.A. Epstein and N.E. Steenrod, Cohomology operations, Ann. Math. Studies 50, Princeton Univ. Press, Princeton, 1962.

[5] M. Gerstenhaber, The cohomology structure of an associative ring, Ann. Math. 78 (1963), 267-288.

[6] M. Gerstenhaber, On the deformation of rings and algebras, Ann. Math. 79 (1964), 59-103.

[7] M. Gerstenhaber and S.D. Schack, Algebras, bialgebras, quantum groups, and algebraic deformations, Contemp. Math. 134 (1992), 51-92.

[8] M. Gerstenhaber and S.D. Schack, Bialgebra cohomology, deformations, and quantum groups, Proc. Nat. Acad. Sci. U.S.A. 87 (1990), 478-481.

[9] G. Hochschild, On the cohomology groups of an associative algebra, Ann. Math. 46 (1945), 58-67.

[10] D.W. Jonah, Cohomology of coalgebras, Mem. Amer. Math. Soc. 82, Amer. Math. Soc., Providence, 1968.

[11] C. Kassel, Quantum groups, Grad. Texts in Math. 155, Springer-Verlag, New York, 1995.

[12] A. Kaygun, Hopf-Hochschild (co)homology of module algebras, Homology Homotopy Appl. 9 (2007), 451-472.

[13] P.S. Landweber, Cobordism operations and Hopf algebras, Trans. Amer. Math. Soc. 129 (1967), 94-110.

[14] J. Milnor, The Steenrod algebra and its dual, Ann. Math. 67 (1958), 150-171. 
[15] S. Montgomery, Hopf algebras and their actions on rings, CBMS Regional Conference Series in Math. 82, Amer. Math. Soc., Providence, 1993.

[16] S.P. Novikov, Methods of algebraic topology from the point of view of cobordism theory, Izv. Akad. Nauk SSSR Ser. Mat. 31 (1967), 855-951.

[17] B. Parshall and J.P. Wang, On bialgebra cohomology. Algebra, groups and geometry, Bull. Soc. Math. Belg. Sér. A 42 (1990), 607-642.

[18] M. Sweedler, Hopf algebras, W.A. Benjamin Inc., 1969.

[19] D. Yau, Deformation of algebras over the Landweber-Novikov algebra, J. Algebra 298 (2006), 507-523.

[20] D. Yau, Cohomology and deformation of module-algebras, J. Pure Appl. Alg. 209 (2007), 781-792.

[21] D. Yau, Brace operations and Deligne's Conjecture for module-algebras, preprint, arXiv:math.KT/0607629.

Donald Yau dyau@math.ohio-state.edu

Department of Mathematics, The Ohio State University at Newark, 1179 University Drive, Newark, OH 43055, USA 